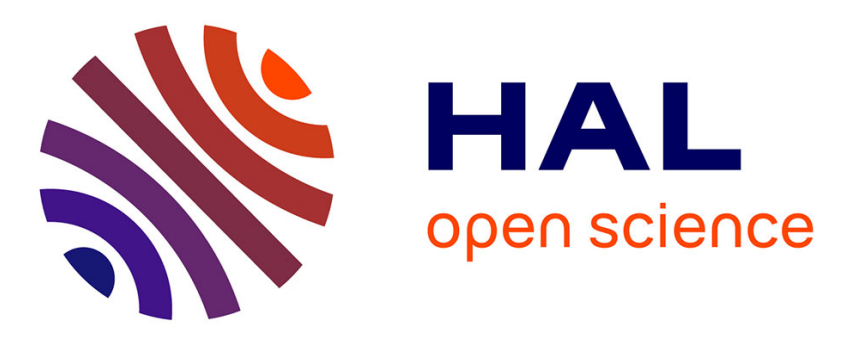

\title{
New Perspectives for the Future Interoperable Enterprise Systems
}

Hervé Panetto, Milan Zdravković, Ricardo Jardim-Gonçalves, David Romero, J. Cecil, István Mezgár

\section{- To cite this version:}

Hervé Panetto, Milan Zdravković, Ricardo Jardim-Gonçalves, David Romero, J. Cecil, et al.. New Perspectives for the Future Interoperable Enterprise Systems. Computers in Industry, 2016, Special Issue: "Future Perspectives on Next Generation Enterprise Information Systems: Emerging Domains and Application Environments", 79, pp.47-63. 10.1016/j.compind.2015.08.001 . hal-01142747

\section{HAL Id: hal-01142747 \\ https://hal.science/hal-01142747}

Submitted on 5 Jun 2015

HAL is a multi-disciplinary open access archive for the deposit and dissemination of scientific research documents, whether they are published or not. The documents may come from teaching and research institutions in France or abroad, or from public or private research centers.
L'archive ouverte pluridisciplinaire HAL, est destinée au dépôt et à la diffusion de documents scientifiques de niveau recherche, publiés ou non, émanant des établissements d'enseignement et de recherche français ou étrangers, des laboratoires publics ou privés. 


\title{
New Perspectives for the Future Interoperable Enterprise Systems
}

\author{
Hervé Panetto $^{1,2 *}$, Milan Zdravkovic ${ }^{3}$, Ricardo Jardim-Goncalves ${ }^{4}$, \\ David Romero ${ }^{5,6}$, J. Cecil ${ }^{7}$, István Mezgár 8,9
}

\author{
${ }^{1}$ Research Centre for automatic Control (CRAN UMR 7039), University of Lorraine, France \\ ${ }^{2}$ Research Centre for automatic Control (CRAN UMR 7039), CNRS, France, herve.panetto@univ- \\ lorraine.fr \\ ${ }^{3}$ Faculty of Mechanical Engineering, University of Nis, Serbia, milan.zdravkovic@gmail.com \\ ${ }^{4}$ New University of Lisbon, UNINOVA, Portugal, rg@uninova.pt \\ ${ }^{5}$ Tecnológico de Monterrey, Mexico, david.romero.diaz@gmail.com \\ ${ }^{6}$ Griffith University, Australia \\ ${ }^{7}$ School of Industrial Engineering, Oklahoma State University, USA, J.Cecil@ okstate.edu \\ ${ }^{8}$ Department of Manufacturing Science and Technology, Budapest University of Technology and \\ Economics, Hungary \\ ${ }^{9}$ Computer and Automation Research Institute, Hungarian Academy of Sciences, Hungary, \\ mezgar.istvan@sztaki.mta.hu
}

\begin{abstract}
:
The rapid changes in today's socio-economic and technological environment in which the enterprises operate necessitate the identification of new requirements that address both theoretical and practical aspects of the Enterprise Information Systems (EIS). Such an evolving environment contributes to both the process and the system complexity which cannot be handled by the traditional architectures. The constant pressure of requirements for more data, more collaboration and more flexibility motivates us to discuss about the concept of Next Generation EIS (NG EIS) which is federated, omnipresent, model-driven, open, reconfigurable and aware. All these properties imply that the future enterprise system is inherently interoperable. This position paper presents the discussion that spans several research challenges of future interoperable enterprise systems, specialized from the existing general research priorities and directions of IFAC Technical Committee $5.3^{1}$, namely: context-aware systems, semantic interoperability, cyber-physical systems, cloud-based systems and interoperability assessment.
\end{abstract}

\section{Introduction}

Increasing diversity of the environment in which enterprises are collaborating with its customers and suppliers has established interoperability as a scientific challenge of major importance. The need to address this challenge becomes even more important when considering that new paradigms such as Internet of Things and Cyber-Physical Systems have introduced new identifiable resources with communication and processing capability and have contributed to significantly increased complexity of the technical environment in which above mentioned collaborations take place.

Although industry has responded to the interoperability challenges with the development of collaboration interfaces and integration mechanisms, such development may become unsustainable with the rapid growth in the variety of system architectures. The traditional approaches to achieve interoperability have targeted facilitating negotiation between systems to enable meaningful and purposeful interoperations. When this negotiation is carried out in processes where two systems attempt to understand each other's interfaces, then capability to interoperate is dependent on its "capability to understand". This consideration can be extended to include the capability to understand various types of stimulus or data from a (including raw data, perceived physical phenomenon, a message from another system or a social media event).

The need for continued research in Enterprise Integration and Interoperability has to be underscored along with identifying the developing trends and issues of relevance to the broader domain of Enterprise Information Systems (EIS). It is important to recapitulate the Grand Challenges defined in Panetto and Molina (2008), which are still valid. This earlier identification of Grand Challenges emphasized the importance of Collaborative Networked Organizations, the adoption of Enterprise Modelling techniques and Reference Models (while emphasizing model reuse), the importance of addressing Enterprise and Process Models Interoperability, the need to develop methods to support Verification, Validation, Qualification and Accreditation of Enterprise Models.

\footnotetext{
${ }^{1}$ IFAC Technical Committee 5.3 « Enterprise Integration and Networking », http://www.ifac-tc53.org
} 
It is also important to note that recent advances in information and communication technologies (ICT) have allowed enterprises to move from highly data-driven environments to a more cooperative information/knowledge-driven environment (Panetto and Cecil, 2013). The supervision, monitoring, adaptation and control of the enterprise systems dynamics and of the eco-system of network(s) they are operating are the requirements that guide the design of an effective management framework. This is especially true when recognizing the fact that new ICT governance models will continue to emerge; this in turn will lead to continued evolution of their information systems and infrastructure accordingly (Jardim-Goncalves and Grilo, 2010).

According to Svejvig and Møller (2012), while first generation EIS were application-centric with not necessarily integrated business rules, second generation EIS can be considered to be data-centric and driven by the integrated databases. Third generation EIS focus on business processes with appropriate process management architectures, supported by integrated systems. Fourth generation EISs (current one) are human-centric and they are driven by new human-machine interfaces, with challenges for standards, security, transparency of control, user simplicity, rights management, IT and business working in a cooperative environment. According to authors, fifth generation EIS (emerging) can be considered things-centric and driven by the digitalisation and integration of "things" into a cyber-physical environment, with challenges for interfaces and communication platforms as well as service-oriented architectures. Then, next-generation EIS (NG EIS) can be considered everythingcentric and driven by creating interplay networks of people, data, things and services, with challenges for achieving secure openness and capability to interoperate of and with all entities (Table 1).

Table 1. Enterprise Information Systems Generations (Extended from Svejvig \& Møller, 2012)

\begin{tabular}{|c|c|c|c|c|c|c|}
\hline \multirow{2}{*}{$\begin{array}{c}\text { EIS } \\
\text { Generation }\end{array}$} & 1 & 2 & 3 & 4 & 5 & 6 \\
\hline & $\begin{array}{c}\text { Application } \\
\text { Centric }\end{array}$ & $\begin{array}{c}\text { Data } \\
\text { Centric }\end{array}$ & $\begin{array}{l}\text { Process } \\
\text { Centric }\end{array}$ & $\begin{array}{l}\text { Human } \\
\text { Centric }\end{array}$ & $\begin{array}{l}\text { Things } \\
\text { Centric }\end{array}$ & $\begin{array}{c}\text { Everything } \\
\text { Centric }\end{array}$ \\
\hline Timeline & $\begin{array}{c}\text { Around } \\
80 \text { 's }\end{array}$ & $\begin{array}{l}\text { Around } \\
90 \text { 's }\end{array}$ & $\begin{array}{c}\text { Around } \\
00 \text { 's }\end{array}$ & $\begin{array}{l}\text { Around } \\
10 \text { 's }\end{array}$ & $\begin{array}{c}\text { Around } \\
15 \text { 's }\end{array}$ & $\begin{array}{l}\text { Around } \\
20 \text { 's }\end{array}$ \\
\hline $\begin{array}{c}\text { Iconic } \\
\text { Technology }\end{array}$ & MRP & ERP & BPM & HMI & SENSORS & DEVICES \\
\hline $\begin{array}{c}\text { Technology } \\
\text { Drivers }\end{array}$ & Databases & $\begin{array}{c}\text { DBMS, } \\
\text { Client-Server } \\
\text { Architecture }\end{array}$ & $\begin{array}{c}\text { Internet, } \\
\text { SOA }\end{array}$ & $\begin{array}{c}\text { Semantic } \\
\text { Networks, } \\
\text { Intelligent } \\
\text { Social Media, } \\
\text { Cloud } \\
\text { Computing, } \\
\text { Virtual and } \\
\text { Augmented } \\
\text { Reality }\end{array}$ & $\begin{array}{c}\text { Smart } \\
\text { Things, } \\
\text { Wireless } \\
\text { Sensor } \\
\text { Networks, } \\
\text { Big Data, } \\
\text { Service } \\
\text { Science, } \\
\text { Cloud } \\
\text { Computing }\end{array}$ & $\begin{array}{c}\text { Real Plug \& } \\
\text { Play Systems, } \\
\text { Open and } \\
\text { Trusted } \\
\text { Platforms, } \\
\text { Trustworthy } \\
\text { Infrastructures, } \\
\text { Interoperability } \\
\text { Service Utility }\end{array}$ \\
\hline $\begin{array}{l}\text { Deployment } \\
\text { Environment }\end{array}$ & \multicolumn{2}{|c|}{ Local Systems } & \multicolumn{2}{|c|}{ Digital Systems } & \multicolumn{2}{|c|}{ Cyber-Physical Systems } \\
\hline $\begin{array}{c}\text { Business } \\
\text { Challenge }\end{array}$ & Efficacy & Efficiency & Effectiveness & Resilience & Sensitiveness & Proactiveness \\
\hline $\begin{array}{l}\text { Organisational } \\
\text { Challenge }\end{array}$ & $\begin{array}{c}\text { Support of } \\
\text { Departments }\end{array}$ & $\begin{array}{l}\text { Support of } \\
\text { Enterprises }\end{array}$ & $\begin{array}{l}\text { Support of } \\
\text { Supply } \\
\text { Chains }\end{array}$ & $\begin{array}{l}\text { Support of } \\
\text { Social } \\
\text { Networks }\end{array}$ & $\begin{array}{l}\text { Support of } \\
\text { Sensor } \\
\text { Networks }\end{array}$ & $\begin{array}{l}\text { Support of } \\
\text { Interplay } \\
\text { Networks }\end{array}$ \\
\hline $\begin{array}{l}\text { Technology } \\
\text { Challenge }\end{array}$ & \multicolumn{3}{|c|}{ Systems Integration } & \multicolumn{3}{|c|}{ Systems Interoperability } \\
\hline $\begin{array}{l}\text { Knowledge } \\
\text { Challenge }\end{array}$ & $\begin{array}{c}\text { Structured } \\
\text { Data }\end{array}$ & $\begin{array}{r}\text { Integra } \\
\text { Data }\end{array}$ & Dynar & Data & Time Data & $\begin{array}{c}\text { Inferred } \\
\text { Data }\end{array}$ \\
\hline
\end{tabular}

The properties of these future NG EISs have been identified based on the synthesis of the different works, mainly done within Future Internet Enterprise Systems (FInES) cluster of projects, supported by the European Commission. In its recently published research roadmap (FInES, b), FInES cluster identified the ICT solutions and socio-technical systems aimed at supporting the emerging future enterprises that will largely operate over the Future Internet, including also enabling technologies. The following properties of NG EIS are identified:

Omnipresence: Omnipresence refers to being present everywhere. In an EIS context, this refers to the implementation or existence of EIS on a wide range of computing and other platforms. Traditionally, EIS have been implemented on networked computers and the web for various application contexts. Recently, the advent of 'thin clients' such as tablets, smart phones, smart watches and other devices has provided the basis for the continued evolution of deployment scenarios for next generation information systems. As the computing power of these thin clients and devices increase, EIS' can be run on such clients (through cloud or other technologies) as well can be implemented through integrating an array of distributed components deployed from various locations in the world. 
Model-driven architecture: The behaviour of Next Generation Systems will be driven by advanced information based models (from distributed locations) which can control the functionality of the corresponding collaborative enterprises; this can radically influence the accomplishment of a given product development life-cycle or any other non-engineering activity or set of activities. For these reasons, the architectures of such systems can be described as being 'model- driven'. By adopting a different model or modifying an existing model, the functional behaviour of a given enterprise can be modified (or 'controlled' to address changing customer, environment, industry or other requirements.

Openness: In the EIS context, 'openness' typically refers to technological (and legal) accessibility of software entities, artefacts and distributed collaborative processes based on knowledge sharing between peers. In broader contexts, openness can also refer to transparent organisational structures. Such practices can include 'Internal openness', 'external openness' or a hybrid combination of both. Internally, technologies to support such openness can include co-creation environments and tools within the daily functioning of an organization. Externally, openness can include adoption of crowdsourcing, and open innovation market based approaches. Such characteristics will dramatically impact both inter-organisational and intra- organisational ways to creating new products and processes while providing robust support to relevant ecosystems of various components and agents; an example is the ecosystem surrounding Google's Android OS [ECIS 15]. With the advent of cyber-physical frameworks, an important outcome of adopting openness is that there will be a greater emphasis on information and information driven models which can interface with on-demand physical facilities and resources. For such openness to become a reality, the issue of semantic interoperability needs to be effectively addressed to support collaboration across platforms and applications.

Dynamic Re-configurability: Re-configurability refers to the ability to function in a different manner to perform the same function or accomplish a different set of functions using the same or improved set of resources. An essential capability to achieve this will be the availability of seamless interfaces to 'process' and 'activity' related information models which can be modified to reflect such new functionalities, processes or activities. Apart from being model driven, such NG EIS can also access and use specific functional components (for various services in an enterprise's life cycle); if necessary, it can re-configure itself based on a different model and then implement various functions according to this new model.

Multiplicity of Functional Identities: NG EIS systems in the future will be capable of different functional identities based on given application, process and domain contexts. For instance, in a certain context, such a system can be a complex software agent performing a specific task in a social networking application; in another context, it can be a collaborative process integration system capable of coordinating advanced engineering life cycle activities.

Semantic and Sensory Awareness: Awareness refers to recognizing lower level process changes (through sensors and other devices) as well as higher level semantic constructs which are both essential to achieving seamless functional integration across multiple contexts and platforms. NG EIS will need to be able to accept, process and perform various functions based on lower level sensory inputs from distributed or co-located devices. At a higher level, such EIS will need to understand and resolve semantic interoperability bottlenecks across multiple platforms and collaborating components.

Advanced Reasoning and Computing Flexibility: NG EIS will be increasingly required to function beyond the 'normal' reasoning and computing capabilities found in typical enterprise systems for engineering and business applications. They will need to be capable of fuzzy reasoning depending on incomplete data/information/knowledge available for various contexts.

The list of properties implies that interoperability is foreseen as an inherent capability of the NG EIS. It facilitates forming ad-hoc collaborations; it uses the correspondences between the different models and enables their use by the core execution environments; it integrates data, information and knowledge resources and services; it enables a federation of NG EIS functions to its smart objects, agents and avatars. 


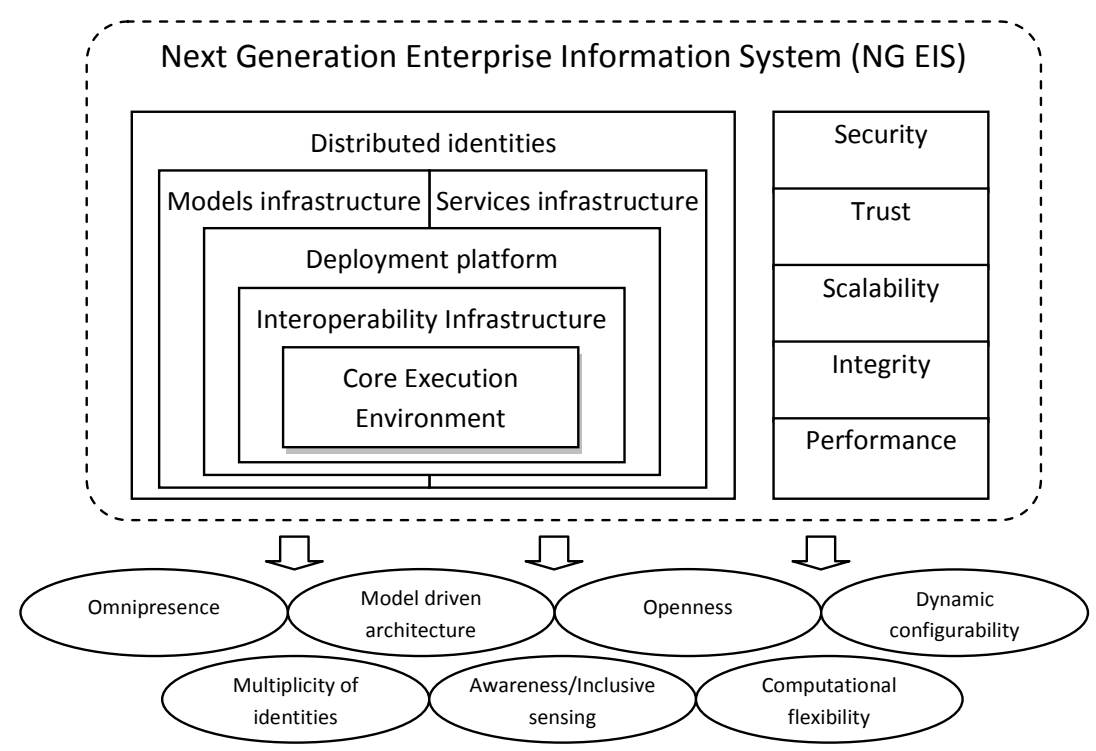

Fig. 1. Abstract Architecture of NG EIS

The listed properties are used to propose the generic abstract architecture of the NG EIS, as illustrated on Fig. 1.

Hence, research challenges are categorized based on the consideration of the identified properties of NG EIS in context of the past work of IFAC TC5.3. The specific challenges were identified in context of the conventional EIS lifecycle (with disregarded development phase, considered as non-relevant), as it is illustrated on Fig. 2.

Therefore, this paper describes challenges, trends and issues that must be addressed to support a new generation of scientific-based and technological solutions for facilitating the collaboration of existing enterprise systems while addressing the sustainability requirements of collaborative environment itself, and of their products and processes. The following challenges for the development of NG EIS are identified: context-aware systems, semantic interoperability, cyber-physical systems, cloud-based systems and interoperability assessment. The progress towards resolving the respective issues will then create opportunities for new organisational forms and business models that will transform the conventional enterprise (see Fig. 2).

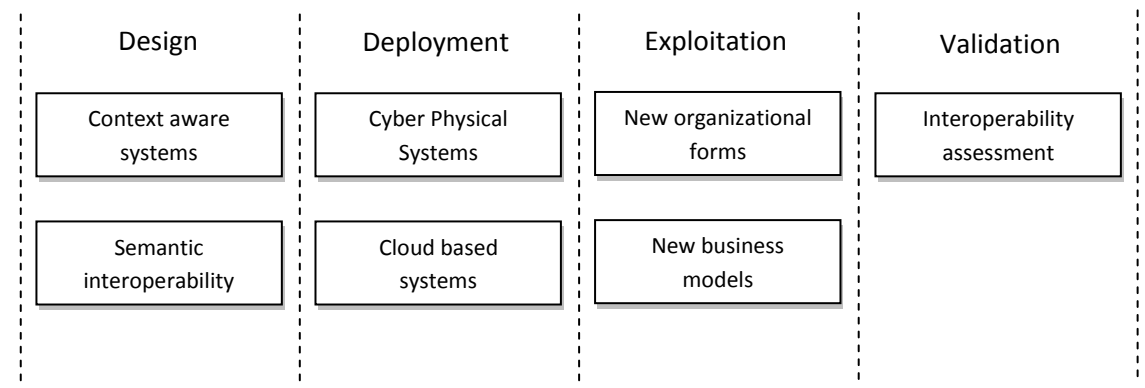

Fig. 2 The Research Challenges for NG EIS

In Section 2, we explore the above mentioned challenges and discuss on their possible contribution to the development of NGEIS. Then, in Section 3, an overview of the tools, technologies, methodologies and knowledge resources for resolving these challenges, so-called enablers of NGEIS are presented. Finally, the Section 4 discusses about the potential impact of the listed technologies to the transformation of the conventional enterprises and their information systems to the future, Internetbased business endeavours.

\section{Research Challenges for the Future Interoperable Enterprise Systems}

This section elaborates on the identified research challenges for NG EIS (see Fig. 2), namely, contextaware systems, semantic interoperability, Cyber-Physical Systems, Cloud-based systems and interoperability assessment. In addition it is discussed possible impact of resolving these challenges, throughout the contribution of the respective scientific topics to the development of NG EIS. 


\subsection{Context-Aware NG EIS}

Today, enterprises should be addressed as complex adaptive systems, stimulated by extensive and resilient sensorial capabilities that are able to detect physical and virtual stimulus, recognising the application context of specific operational situations and responding and/or reacting accordingly (Jardim-Goncalves et al., 2013). To achieve such capabilities, the concept demands a novel framework with normalized reference models, formal methods, standardized architecture and open platform and tools, model and process adaptation and morphisms for data and model transformation (Agostinho et al., 2011). Such assets will enable the enterprises with the capability for global application context awareness and with the faculty to perceive their holistic actual operational status in a real-time and anticipate future decisions by using multi-dimensional and multi-modal information.

Context-awareness brings the ability to assume connectivity among everything, anywhere and anytime, independently of the means and providers. The NG EISs will receive a real-time physical (e.g., through networks of physical sensors) and virtual (e.g., through data analytics from cloud or social media sources) information about enterprises operating environment, which is 'global' by nature, including tweets, sensing information, RFID or GPS data, to mention only a few examples (Frankston, 2009). Real-time information from both the physical and the virtual surroundings of the enterprise is fed constantly into its decision making process, whether the latter is still controlled by the 'subjects' (i.e. the human beings) or by the 'objects' (i.e. the various sorts of Internet of Things-enabled artefacts) (FInES, b) (Svejvig and Møller, 2012).

Cyber-Physical Systems theories have been taken as a reference to move beyond the state of the art in this area. They embrace hierarchical design for modelling complex and distributed systems that require a consistent supporting framework, together with re-use of previous developments on standards and meta-standard architectures for an effective and systematic approach to deal with complexity. Embedded in the system's design, semantic interoperability based on the ability to deal with ontologies and taxonomies has to be considered (Ferreira et al., 2013).

Removing the complexity, by detaching the context models from the native NG EIS architecture will contribute to their increased flexibility and hence, to their dynamic configurability. Now, these context models will become a part of loosely integrated (by logical correspondences, forming the unification models themselves) NG EISs models infrastructure, from which behavioural models of each of the NG EISs digital identities will be possible to infer.

Current main challenges for the NG EIS are within dynamic configurability, competitive and customer Intelligence, intelligent reconfiguration, automatized categorization of ontological structures, transient analysis and adaptive services. This challenges conduct to a set of envisaged solutions in a short, medium and long term, i.e., IoT-facilitated intelligent response of the enterprises to their environment (short), Implementing virtual and physical sensing capabilities and connecting them to the digital capabilities of the Future Internet (medium), Inclusive (multiple dimensions and stakeholders) sensing (long), and Consolidation of efforts to create large possibilities of innovative business (long).

\subsection{Semantic Interoperability as a Capability of NG EIS}

Semantic interoperability of systems is foreseen as an approach to establish the universal and unconditional interoperability. Namely, it is expected to reduce the volume of pre-conditions/preagreements needed to be fulfilled/established before two systems can effectively interoperate. Unfortunately, it seems that the expectations from the semantic interoperability are failing beyond the limits of the academic exercises. For example, the research community failed to achieve effective automatic reconciliation of semantic heterogeneities. In practice, this reconciliation is done manually. Hence, it is error-prone, labour intensive and thus, inefficient. It is common that $60-80 \%$ of the resources in data sharing projects are spent on addressing the issues of semantic heterogeneities (Doan et al., 2004).

The semantic interoperability goes beyond mere data exchange and deals with its interpretation, in order to facilitate interoperability based on the interpreted meaning. The notion of interpretation implies that some kind of intelligence is needed by the systems so to understand the information that is being transmitted. In the traditional research of semantic interoperability, the process of understanding is reduced to the process of reasoning about the transmitted concepts. The development of intelligent agents and related concepts inspired the work on defining the interoperability capability by using anthropomorphic perspective which goes far beyond simple reasoning. In fact, this complex capability is comprised of system's abilities to sense, perceive its environment, make an intelligent decision on the response to a perceived meaning of the stimuli and articulate this response (Zdravković et al., 
2015). The assumption is based on analogies with the human communication process, which is today considered as interplay of four physiological and psychological groups of processes: sensation (physiological), perception, cognition and articulation (Littlejohn and Foss, 2010). Hence, the interoperability capability of one NG EIS is constituted by the corresponding attributes of awareness, perceptivity, intelligence and extroversion. These attributes are further decomposed and formally defined in Zdravković et al. (2014b). Although the cited work is one step in this direction, the lack of unified interoperability theory is considered as a significant obstacle for innovation in the semantic interoperability research arena.

While the awareness property of systems is already well developed in the research typically associated to the area of CPS, achieving the perceptive system is still a huge challenge. Perceptivity is a capability of a NG EIS to assign a meaning to the observation from its environment or from within itself. It is based on the access to a wide range of perceptual theories or sets, consisting of different ontologies representing the domain knowledge, but also motivational aspects of communication, e.g. different problem or application ontologies. Despite the data explosion and rising use of semantic technologies for its representation (open linked data), the underlying semantic structures (ontologies) are still fragmented and uncorrelated. This is considered as a main challenge in forming the complex perceptual sets, needed to implement perceptive systems.

Based on the perception, the NG EIS should be able to decide on the consequent action. This decision is a result of a cognitive process, which consists of identification, analysis and synthesis of the possible actions to perform in response to the "understood" observation. The intelligence also encompasses assertion, storing and acquisition of the behaviour patterns, based on the post-agreements on the purposefulness of the performed actions.

\subsection{Cyber-Physical NG EIS}

A cyber-physical oriented enterprise system (CPES) can be described as a system comprising of a heterogeneous mix of software and physical components which support collaborations to achieve a functional objective of creating new products or providing services in a range of domains. Other researchers have outlined cyber-physical systems as a system of collaborating computational elements controlling physical entities (Wang et al., 2013). In a process engineering context, such cyber- physical systems can be viewed as an emerging trend where software tools interface/collaborate with physical devices to accomplish a variety of activities ranging from sensing, monitoring to advanced assembly and manufacturing.

The CPS frameworks and architectures make possible to embed ICT systems in all types of artefacts making our daily-used systems and objects, such as cars, factories, hospitals and personal devices "smarter", more intelligent, more energy-efficient and more comfortable. CPS enable a new generation of "smart" systems, through the convergence of networking and information technologies with manufactured products, engineered systems and associate services. Often provided with control, monitoring and data gathering functions, CPS have to comply with essential requirements like safety, privacy, security and very low power consumption, together with size, usability and adaptability constraints.

Thus, CPSs become core, native components of NG EIS, and the key technical enablers of their omnipresence. As such, they need to inherit all their properties and implement those on the deployment level. Namely, they will implement technical protocols and standards for (flexible) processing, communication (semantic interoperability) and persistence (cloud). These high-level protocols and standards will not only enable further development of the NG EIS-related paradigms; they will also contribute to their own de-verticalisation, and hence, to extension of the application base, even to their commoditization. Finally, the development of these protocols and standards will provide important input to further evolution of the existing communication infrastructure towards the paradigm of Next generation Internet.

\subsection{Cloud-based NG EIS}

According to Gartner's forecast, by 2020 the number of smart-phones, tablets and PCs in use will reach about 7.3 billion units while the IoT (Internet-of-Things) will expand at much faster rate, resulting in a number of about 26 billion units at that time (Middleton, 2013). New paradigms, approaches, technologies and devices are needed to be able to handle (generate, transmit, collect, store, process) this epic flow of data in a flexible, reliable and secure way. Cloud computing is one of the main technologies to support the efforts to overcome this extreme data flows. 
Cloud computing is a model for enabling ubiquitous, convenient, on-demand network access to a shared pool of configurable computing resources (including networks, storage, services and servers); the computing resources can be rapidly provisioned with reduced or minimal management effort or interaction with service providers. Cloud-Based Systems (CBS) are flexible, scalable and can operate very economically from the point of users. The essential characteristics of cloud computing are: Ondemand, self-service; Broad network access; Resource pooling; Rapid elasticity; and Measured Service (Mell and Grance 2009),

When taking into consideration the present status, applications, trends and the forecasts on the future of cloud computing, we foresee that the CBS will be the key repository and deployment component of the future NG EIS. However, this assumption holds only if certain specific challenges of CBS are addressed effectively in the future. The main challenges for CBS are: interoperability (inside the cloud; between clouds; between conventional and CBS), security (communication, endpoint and data storage) and privacy (ownership, business and personal data access). Beyond the technical challenges the operational and legal aspects also should be mentioned as specific serious challenges.

The key argument for the assumption that CBS will constitute a key deployment and storage components of the future NG EIS is related to its flexibility and collaboration potential. Cloud computing offers significant financial advantages (pay only for what you use, less in-house IT staff and costs, etc.) while offering high level collaboration possibilities (Buyya et al., 2009). Cloud computing, includes many aspects of computing (from hardware to software) so they can be described by different models. When their service and deployment characteristics are considered, they can be classified into three service models, as:

- $\quad$ Software as a Service (SaaS),

- $\quad$ Platform as a Service (PaaS),

- Infrastructure as a Service (IaaS).

Clouds may be hosted and employed in different styles depending on the use case, respectively the business model of the provider:

- Private cloud,

- Community cloud,

- Public cloud,

- Hybrid cloud,

- $\quad$ Special Purpose Clouds.

The main benefits of this approach are that the users of services do not need to own and manage the capital equipment involved. From financial aspect cloud computing represents a "pay-per-use" business model with lower costs in most cases and this is the biggest advantage for an average user, customer. Other general advantages of cloud computing include, but not restricted to: the massive scale, the homogeneity, the virtualization, resilient computing, low cost software, geographical distribution, service orientation and advanced central security technologies. Security advantages are related to the fact that shifting public data to an external cloud reduces the exposure of the internal sensitive data; cloud homogeneity makes security auditing/testing simpler; clouds enable automated security management and redundancy/disaster recovery are simpler. Advantages in data storage are the automated replication, the data fragmentation and dispersal, provision of data zones (e.g., by country), encryption in storage/in transfer and automated data retention.

\subsection{Interoperability Assessment in and for NG EIS}

Interoperability assessment (often referred as interoperability evaluation) is foreseen as an approach to evaluate, quantitatively or qualitatively, a priori of any interoperation process or a posteriori between existing collaborative enterprise information systems.

As cooperative environment progressively evolve, two (or more) information systems need to interoperate between each other in order to fulfil a given objective determined by the cooperation context. Typically, this cooperation requires that heterogeneous information systems coordinate with each other by sharing information. The interoperability occurs when each one of those systems is able to successfully use the exchanged information. Whether it is related to exchange and use of information, or to carrying out an operation on behalf of another system, this is commonly called interoperation (Whitman et al., 2006). Interoperation is also characterised as the relationship of the exchange and the cooperative use of information (Fisher, 2006). In practice, the achievement of the cooperation depends strongly on the effectiveness of the interoperation between the participating systems. That means that, at least, those systems must be minimally interoperable with each other to complete the global system goal. In this context, assessing the consequence of the lack of interoperability between systems is a critical issue (Yahia et al., 2012) and is considered as one of the 
key challenge for EIS interoperability research today. The dynamicity of the interrelationships is then an issue that imposes new formal definitions. For achieving a given goal, EISs work together and develop different interactions or interoperability relationships between each other. They are autonomous with regards to their own mission and they are heterogeneous with regards to their architecture and content. Current research on interoperability measurement mainly focuses on maturity measures (Guedria et al., 2015) based on a variety of models. Generally speaking, Enterprise Interoperability (EI) maturity can be measured in two ways: a priori where the measure relates to the potential of a system to be interoperable with a possible future partner whose identity is not known at the moment of evaluation, or when the measure relates to the level of interoperability between two (or more) known systems willing to interoperate or a posteriori when the assessment is related to the measurement of the performance of an existing interoperability relationship between two systems (Yahia et al., 2012). Thus, evaluating the interoperability of theses information systems, based on the evaluation of the degree of coupling between processes is a Grand Challenge that would allow evaluating the semantic relationships and identifying the activity replication and overlapping, as well as the aspects related to interaction/cooperation.

\section{Enablers of the Interoperable NG EIS}

Despite the fact that NG EIS synthesizes many very ambitious concepts and technologies that are not yet developed, an analysis of the state of the art in the selected relevant research areas has indicated a potential convergence of the current research efforts towards NG EIS. In this section, these efforts are shortly presented; the respective results are considered as the enablers of the interoperable NG EIS, in each of the designated fields, namely context-awareness, semantic interoperability, cyber-physical systems, cloud computing and interoperability assessment. Then, a short discussion on the specific challenges related to realizing the contribution of the enablers towards realization of NG EIS is presented.

\subsection{Context-Awareness}

Recognised as one of the key challenges for the interoperable NG EISs, the context-awareness property will facilitate their sensorial capabilities, but also it will affect their core design (Bakillah, 2015) (Villegas, 2013) (Zhang, 2013). Thus, the research of context-aware systems will need to span a variety of scientific topics and address different theoretical and practical issues (Jardim-Goncalves, 2013). In this section, we present non-exhaustive list of the relevant domains of interest for that research, considered as the enablers for advancing in achievement of the context awareness of NG EIS. The domains include Ambient connectivity, Dynamic configurability, Systems complexity, Networks and web science, Models based systems and Systems engineering, Competitive and customer intelligence.

Ambient Connectivity: Ambient Connectivity brings the ability to establish connectivity among everything, anywhere and anytime, independently of the means and providers (Monfort, 2012) (Juarez, 2014). The Sensing Enterprise receives real time information about its environment, which is 'global' by nature, with tweets, sensing information, RFID or location data that leverage the sensing capabilities of the enterprise (Agostinho, 2015). Real-time information from both the physical and the virtual surroundings of the enterprise is fed constantly into its decision making process, whether the latter is still controlled by the 'subjects' (i.e. the human beings) or by the 'objects' (i.e. the various sorts of IoTenabled artefacts) (Memon, 2014).

Dynamic Configurability: Dynamic configurability supports a system in how it operates with a great deal of flexibility (Mokhtar, 2012). Since IoT systems are made of thousands of nodes and devices, such as sensors and actuators, their configuration and reconfiguration are of strategic value for the enterprise (Perera, 2014). Dynamic and automatized remote configuration facilities need to be provided for enabling self-management applications to automatically configure the parameters required for the applications and the users, and therefore to continuously adapt to the changing contexts of enterprises' operations.

Systems Complexity: Systems have many structures and relationships. Understanding their interactions is considered a major factor in anticipating and directing the enterprise's behaviour in a current context. Implicit is the view that systems are complex and adaptive (CAS). Preliminary theories have been advanced in specific scientific disciplines, e.g., biology, to explain the importance and evolution of systems complexity (Gharajedaghi, 2011). Researchers have attempted to extrapolate these results to a "general systems theory" to explain the importance and behaviour of systems in several fields of science, and address the emergence, adaptation, evolution, and self-organization of systems. 
Research challenges include interoperability and system behaviour and adaptability; communication, collaboration and coordination facilities; interoperability of digital ecosystems as complex systems of systems (Blair, 2012).

Network and Web Science: The ability and capability of enterprises to understand the collaboration context is closely linked to the ability and capability of enterprises to interoperate in a network, as a representation of objects that are seamless interconnected by some underlying structure that facilitates common understanding (Shadbolt, 2008). Research challenges in this domain include Enterprise Interoperability and the adaptability, management and efficiency of networks, e.g. random networks, scale free networks, small world networks, pervasive networks, social networks, clustered networks; "basic laws" for governing networks, the interoperability of networks, interoperability of digital networked ecosystems (Agievich, 2013).

Model-Based Systems Engineering: Modelling provides a more rigorous method for capturing, design, analysis, verification and validation of information, relevant for inference of the operational context (Brambilla, 2012) (Völter, 2013). This provides a comprehensive understanding between development teams and the other stakeholders, as well as traceability features that facilitate properties, such as backward compatibility, where the enterprises of the Future, enabled with NG EISs should be able to interoperate with non-evolved enterprises (using the conventional EISs) (Diallo, 2011) (Guédria et al., 2015).

System Intelligence: To understand the context in which one system operates means to be aware of the semantics of the information that potentially constitutes that context, including its potentially complex logical relationships. Information science studies the intrinsic properties and characteristics of information, such as how to use mathematics to represent them, how to deal with uncertainty in information, what are the fundamental units of measure, what are useful metrics, how to measure those uncertainties and metrics (Arch-int, 2013) (Rezaei, 2013). Research challenges include the semantic aspects of interoperability, in particular definition of the basic properties and characteristics of information; the meaning and "common understanding" of information objects; the construction, use and dissemination of information objects; comparison between information objects; measurement of information objects; applying methods such as crawling, scraping or data mining to gather information about the surrounding environment (including competitors and markets) as well as knowledge of technological developments (Fortineau, 2014) (Chungoora, 2013) (Vermesan, 2013).

\subsubsection{Specific Challenges of Context-Awareness}

Research on NG EIS is expected to push beyond the state of the art in systematizing the Sensing Enterprise concept in the described fields, resulting in a novel scientific framework with normalized reference models, formal methods, standardized architecture and open platform and tools, able to realize the demanded universality of the sensing capabilities of the enterprise (Jardim-Goncalves et al., 2011). In specific, further research in the above listed specific fields is expected to contribute to achievement of the following capabilities of the future NGEIS, relevant for the context-awareness property:

1. Intelligent reconfiguration of components, for interoperability maintenance of evolutionary networked systems, including learning and adaptability. After identifying the need to solve an interoperability problem, the related systems typically know very little about the necessities required to have the global system completely interoperable (Cretan et al., 2012). A learning process will be designed to support the adaption of the several system network nodes involved, and thus keep the global network interoperable.

2. Automatized categorization of ontological structures. The resolution of this issue aim to provide an automatized development of ontologies from descriptive specifications in non-specialized language, e.g., queries described in natural language, supported by an engine with feedback for the user, with learning and reconfiguration capacities (Danila et al., 2013).

3. Transient analysis. The global interoperable network, as a complex integrated system, will face transients whenever an internal or external "interference" occurs, e.g., update in one of its nodes. Thus, there will be a period of time when the systems' nodes will need to react and readapt before the system becomes again stable and interoperable. The evolution and progressive adaptation of each network system node would be done supported by a systematic study and analysis of the network transients, as single node, clusters, and global network.

4. Harmonization of ontological structures to support the application dynamics and enable adaptability of users' semantic specifications aims at providing the capabilities for the management of mutation of ontologies, by using stochastic methods to support the updates in the representation of concepts and its instances (Gilad, 2008). It embraces semantic 
harmonization and adaptive mapping in dynamic environments, with mediation of semantic conflicts according to the interactions and evolution with the systems with which it interacts.

5. Evolutive systems. Systems able to endure in time will require an embedded set of 'selfproperties' qualified to re-organize, optimize, configure, diagnose and maintain (INCOSE, 2007). Design rules and methodologies for enterprises intervening at earlier conceptual phases will help implementing 'self-properties' embedded engines for the sensing capabilities of the physical and virtual artefacts (Ducq et al., 2012).

\subsection{Semantic Interoperability Capability of NG EIS}

In this paper, the anthropomorphic consideration of the semantic interoperability capability of NG EIS is suggested as a methodological approach to facilitate understanding of the information being exchanged between the systems and their consequent seamless interoperations. Although it is a novel concept, the analysis and synthesis of the literature has shown that many existing efforts can be taken into account as potential enablers for this approach.

Facilitating Awareness: System behaviour related to the awareness of the NG EIS's can be facilitated by using sensor ontologies, several of which have been developed to represent sensors and their behaviour, since 2004 (Compton et al., 2004). The extensive review of these ontologies was done by W3C Semantic Sensor Network Incubator Group (W3C, 2011), with objective to propose the W3C Semantic Sensor Network (SSN) Ontology. SSN Ontology (Compton et al., 2011) is a formal OWL DL ontology for modelling sensor devices (and their capabilities), systems and processes. It is universal in sense that it does not assume a physical implementation of a sensor. Namely, it can be used to describe the process of sensing by the WSN nodes, as well as by the humans. The sensor ontology is a useful asset for achieving self-awareness property. Also, it can be used to formally describe processing of the pre-determined, expected observations and making direct conclusions.

Facilitating Perceptivity: The observational perceptivity is related to computing a percept on basis of a raw sensor data. Some work in this direction has been done (Barnaghi et al., 2012), in terms of identifying patterns from sensor data. These patterns are then translated into abstractions with an abductive logic framework called Parsimonious Covering Theory (PCT) (Reggia and Peng, 1987). PCT uses domain-specific background knowledge to determine the best explanation for a set of observations, namely to link the patterns to semantic descriptions of different relevant thematic, spatial and temporal features. In the subsequent effort, with the objective to provide a formal semantics of a machine perception, Henson et al. developed IntellegO ontology of perception (Henson et al., 2011). IntellegO was made based on the principles of Neisser's Perception Cycle (Neisser, 1976). Neisser considered a perception as a cyclic process, in which the observation of the environment, followed by the creation of the initial percepts, is often affected by the process in which we are directing our attention for further exploration, in order to get more stimuli required for constructing the final percept. In this process, humans generate, validate and consequently reduce the hypotheses that explain their observations. According to IntellegO, based on the observed qualities of the inherent properties of the observed object, a subject creates a number of percepts as parts of the so-called perceptual-theory. Hence, the specific goal of the perception cycle is to generate a minimum perceptual-theory for a given set of percepts. Generation, validation and reduction of the hypothesis that explain the observations are traditionally defined as a process of semantic integration (Noy, 2004). They depend on the perceptual sets to which the NG EIS has access. These perceptual sets comprise of number of high level generic descriptions of reality (upper ontologies) (Gangemi et al., 2002), enterprise domain theories (ontologies) (Fox et al., 1996) (Uschold et al., 1998) (Madni et al., 2001) (Zdravković et al., 2011), local models of NG EISs themselves (local ontologies) (Zdravković et al., 2014a), but also the descriptions of the motivational factors for interoperations, specific circumstances in which they occur.

Facilitating Intelligence: Currently, the Semantic Web stack of languages (RDF/RDFS/OWL) is the most used approach for representation and reasoning of knowledge. However, the limited expressiveness of DL-based languages urges to consider the pragmatic approach which will foresee new representation and inference languages and strategies, both deterministic and non-deterministic, complemented with OWL (e.g. as extension of OWL). The development of Semantic Web Rule Language (SWRL), combining OWL and RuleML for definition of Horn-like rules (Horrocks et al., 2004 ) is one of the examples that reflect this need. Some attempts have been made also to extend OWL with F-Logic rules and defaults (Kattenstroth et al., 2007), Logic Programming (Motik et al., 2006) and others. Furthermore, the realities of the future interoperating systems requirements will need to locate dealing with vagueness and uncertainty at the top of the priorities. Similarly to above, this uncertain and incomplete knowledge need to be embedded into formal, possibly OWL ontologies. Based on Bayesian Network, Costa and Laskey (2006) formally defined a probabilistic ontology and developed 
the OWL extension (PR-OWL), consistent with the former definition. In probabilistic ontology, each axiom is annotated with a probability that can now be computed for each of the executed queries (Bellodi et al., 2011) affecting this axiom. This computation is often based on Bayesian Network inference procedures, e.g. belief propagation or junction tree (Ding and Peng, 2004).

Facilitating Extroversion: Extroversion as a property is considered as a capability of a NG EIS to commit to articulating and performing an action, based on a decision. It reflects its commitment to act socially, namely to actively inform, affect or change its environment, where this engagement is related also to endorsing or denouncing other "things" actions. It also reflects its curiosity, namely the capability to articulate the request for any additional information needed for a complete reasoning during the processes of perception and decision.

\subsubsection{Specific challenges of Semantic Interoperability}

When taking into account the anthropomorphic approach to semantic interoperability, two key issues for enabling interoperable NG EIS are highlighted: lack of unified interoperability theory and lack of systemic methodological approach to engineering the ontology for semantic interoperability.

There already exist several works aiming at filling this gap. It was first addressed in the domain of federated databases, the first to face the problem of semantic heterogeneities. Lee et al. (1996) provided the specification of interoperable system, based on so-called Context Interchange Architecture, by using first-order logic. Later efforts followed mainly from system engineering community. Euzenat (2001) proposed the definition of semantic interoperability based on the model theory. Kalfoglou and Schorlemmer (2004) proposed a formalization of semantic interoperability based on the BarwiseSeligman theory of information flow, arguing for a theoretical framework based on the analysis and implementation aspects of semantic interoperability scenarios. Naudet et al. (2010) introduced system theory as an approach for interoperability formalization and developed the Ontology of Interoperability (OoI) - a meta-model for ontological descriptions of systems, problems and solutions, which can then be inferred for a computer-aided interoperability diagnosis. In a more recent work, Jardim-Goncalves et al. (2013) posed the challenge of developing an Enterprise Interoperability Science Foundation (EISF) with attempt to systematize the Interoperability Body of Knowledge (IBoK). Liao et al. (2015) analysed the state-of-the-art about semantic annotations used to formally represent knowledge attached to enterprise models. Unfortunately, all these efforts developed partial or full theories that remained largely uncorrelated one with another. In addition, further work on validation of these propositions is needed in order to establish the most relevant conceptualizations.

The number of ontologies, addressing different domains, realities, applications and problems, produced by the scientific community today is growing extensively. Unfortunately, many of these ontologies are isolated, without an ambition to extend or complement existing work. In addition, the approaches to conceptualizing domains vary significantly from one work to another (Lezoche et al., 2012), posing the need to strengthen and further promote the methodological base for ontology engineering, already defined by the influential works of Gruber (1991) and Guarino (1997). When taking into account the anthropomorphic approach to defining the semantic interoperability, proposed in this paper, and in context of methodological approach to ontology engineering, authors propose the "human" ontology continuum (see Fig. 3) as a set of future ontological frameworks, acting as perceptual theories.

According to this, the interoperable (receiving) NG EIS needs to exhibit the domain knowledge, empathy, situational and environmental awareness. Each of these communication capabilities correspond to the representation of the models that facilitate those capabilities, namely, the domain ontologies $\left(\mathrm{O}_{\mathrm{D}}\right)$, problem ontologies $\left(\mathrm{O}_{\mathrm{P}}\right)$ and ontology of emotions $\left(\mathrm{O}_{\mathrm{E}}\right)$. Then, the perceptual sets are formed as schemas in which the different concepts from the above ontologies are logically related between themselves. Layering of domain and problem representation models reflects the paradigm of separation of domain and task-solving knowledge (Gangemi, 2005) and assumes their mutual independence (Guarino, 1997). 


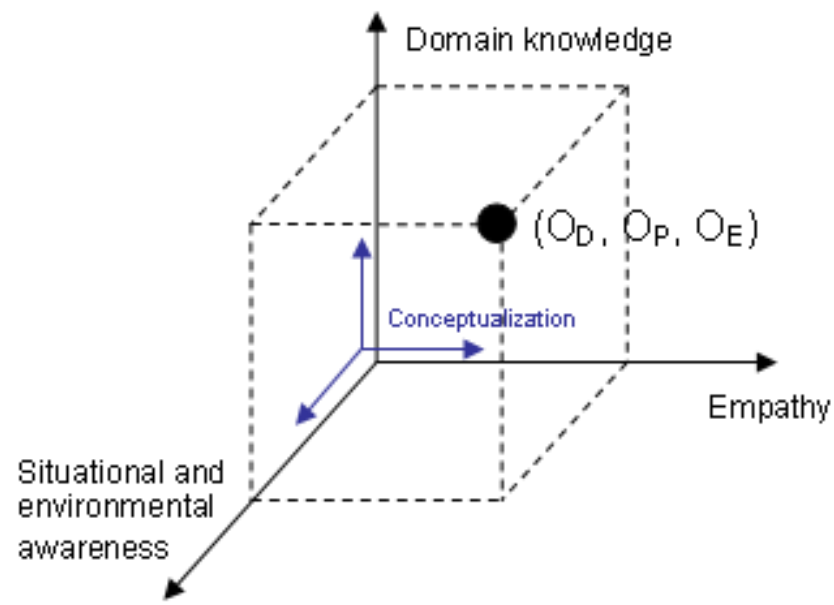

Fig. 3. "Human" Ontology Continuum

Hence, problem or task ontologies are often formed to formalize context-independent, problem solving knowledge. Such ontologies facilitate the situational awareness of the communicating entities. It is also important to highlight that the communication between two NG EISs takes place in some general context, which is not by any means affected by this communication, nor related to it. Environmental conditions and noise are examples of such a case. They change the way one message is articulated and consequently, sensed and perceived. Hence, the perceptual sets must also take into account the formal descriptions of these conditions. In fact, the NG EISs need to be capable to continuously and in a real time: 1) sense these conditions; 2) create and maintain their formal descriptions; 3) create and maintain correspondences of these formal descriptions with "internal" perceptual sets. Emphatic aspect of one perceptual set is related to the capability of NG EIS to interpret and classify emotional states of the information sender and thus to evaluate its message, accordingly (for example, its urgency). Representation and modelling of emotions has become a research challenge with appearance of the affective computing paradigm (Piccard, 2003), that deals with building machines that recognise, express, model, communicate, and respond to emotional information. The emphatic capability of the NG EIS can be enriched by enabling the systems to automatically recognise emotions from structured or unstructured text (Wu et al., 2006).

\subsection{Cyber-Physical Systems and Next-Generation Internet as Enablers of NG EIS}

While semantic interoperability as an inherent capability of NG EIS facilitate context-awareness, the Cyber-Physical Systems are considered as future infrastructures that will host NG EIS, over nextgeneration Internet.

Cyber-physical systems (CPS) are in contrast to traditional embedded systems which deal with stand-alone devices (Kanduri et al., 2013). Embedded systems can be viewed as a pre-cursor generation of cyber-physical systems which can be found in various fields ranging from medicine to transportation systems. An overview of CPS research is provided in Kyoung-Dae and Kumar (2012) including a review of theoretical results on modelling and analysis of dynamic behaviour of CPS. In Woochul et al. (2012), three key properties of CPS is highlighted including safety, security and sustainability. Typically, CPS applications involve sensors monitoring a specific environment and providing feedback to a centralized node; such approaches can be used in medical monitoring, distributed robotics and advanced manufacturing. Although the CPS is a new research field, a review of literature indicates growing trend of implementations of cyber-physical systems. One such application is MITs Distributed Robot Garden where a group of robots services a garden (Cartel 2015). Another application involves calculating fastest routes for taxis in Boston based on traffic information (Enikov and Lazarov, 2001). The general framework for implementing cyber-physical approaches can also serve as a conceptual framework to create advanced and complex implementations in the context of Smart Cities and Internet of Things (IoT). In Wang and Xu (2013), the design aspects of a typical cyber-physical system, including computational modelling and implementation are discussed.

Clearly, massive use of CPS will generate extensive amount of data and Internet traffic. Current infrastructures do not exhibit the performance that matches the respective requirements.

The basic Internet protocol is now 35 years old and the Internet scale has increased by many orders of magnitude. There have been hundreds of additional protocols and extensions, which make its management more and more complex. However, the novel societal and commercial usages are 
continuing to push the original Internet architecture to its limits; it has steered the use of the Internet into directions which were not initially anticipated. It has also resulted in posing demanding technological and policy challenges security, mobility, heterogeneity, complexity, etc. will require a radical redesign or change in the architecture of the current Internet. This is the underlying motivation to design the next Internet (EU FIRE). Recent global initiatives involving the design of the next generation of Internet are underway which can address the need to develop agile, high gigabit networks. Among these are the GENI (Minnick, 2013), FIRE, US Ignite, Fed4FIRE and other initiatives involving US, EU, Japan, Korea, India, China and other countries.

\subsubsection{Specific Challenges referring to Cyber-Physical Systems}

The medium and long term challenges in cyber-physical based EIS include breakthroughs in dependent technologies as well as in addressing key issues related to sustainability and predictability.

In Kwei-Jay and Panahi (2010), a real time service oriented architecture (SOA) is outlined to support sustainability and predictability in cyber-physical systems. In Gobinath and Cecil (2005) and Cecil and Jones (2014), the adoption of an integrated strategy involving use of virtual (cyber) and physical resources for advanced manufacturing is outlined. The need to develop broader frameworks to support such cyber-physical interactions has also been recognised; the benefits of such approaches in tele-medicine are discussed in Cecil et al., (2013). The creation of information centric frameworks to support modelling, simulation and exchange among cyber-physical entities is outlined by other researchers. In the context of advanced manufacturing, the adoption of such an information framework is discussed for the field of micro assembly by Cecil et al. (2013). In Cecil (2015), the preliminary design of a cyber-physical framework for advanced manufacturing (based on emerging GENI technologies) is outlined. In this application, users can propose, compare, analyse, plan and finally assembly micro/meso scale devices. An assortment of cyber resources available through a cloud based infrastructure which has been built using the Global Environments for Network Innovation (GENI) framework and as part of a US Ignite initiative. In Mayyas et al. (2009), a group based programming abstraction called a 'Bundle' is discussed; sensors (such as in cell phones) and sensor based networks are viewed as a foundation for developing new CPS infrastructure. The Bundle abstraction can be used to extend these group based abstractions to support devices such as PDA's and laptops.

Cyber-Physical Systems are highly dynamic in nature in terms of the computing resources and the related physical processes. During runtime, the availability of the various entities can change substantially due to noise, failures, mobility, etc. The supporting network layers for such systems can be unstable. Current network middleware are not designed to handle such dynamic aspects of CPS. In Vicaire et al. (2012), a middleware architecture is proposed which enables timely and reliable sensor data dissemination under unpredictable CPS environments. CPS software is usually built using programming abstractions with little or no temporal semantics. In Sztipanovits et al. (2012), a programming model called PTIDES is outlined that serves as a coordination language for model-based design of distributed CPS; PTIDES can function as a coordination language by controlling components that may be designed and specified using different formalisms.

The long term challenges in CPS based frameworks involve the gradual deployment of the Next Generation of Internet globally. Currently, the next Internet is only accessible to a limited number of users in academia and industry in the US, EU, Korea, Australia, Japan, India, and China. Within the next decade, the newer Internet based on SDN needs to become available to the general public including industrial and other organizations.

Other challenges which need to be addressed in the long term include modelling CPS systems, addressing semantic interoperability issues to support seamless data/information exchange as well as innovative ways to model functionality and implementation architectures to support the realization of CPS based EIS. In Banerjee et al. (2012), the challenges of modelling cyber-physical systems are emphasized including concurrency, sensitivity and heterogeneity. Several modelling technologies are discussed including heterogeneous and concurrent models of computation (MoCs), hybrid system modelling and simulation, joint modelling of functionality and implementation architectures. The need to develop methods and tools for design and analysis of CPS is emphasized in Derler et al. (2012). It also delineated a framework to support the design, simulation and verification of such cyber-physical systems.

In general, the various components of a next generation EIS embracing cyber-physical principles include the following:

- Heterogeneous mix of software or cyber resources (including software tools, modules, etc.) to support the life cycle activities for a given virtual enterprise. 
- Assortment of physical resources including sensors (cameras, bar code readers, RFID based devices which provide feedback to the distributed VE partners), the physical equipment (including manufacturing equipment, material handling mechanisms such as conveyors, robots, etc.).

- Cyber-infrastructure including Next Generation computing networks which will provide the communication mechanisms for collaboration among the distributed cyber-physical resources.

- Software based enterprise level managers and modules which initiate, monitor, integrate and ensure the achievement of the functional objective (which can be manufacturing, engineering, service, social or administrative in nature).

\subsection{Cloud Computing as enabler of Interoperable NG EIS}

Cloud based technologies have the potential to support and implement cyber-physical frameworks, approaches and implementations.

Some of the most critical components of cloud-based systems are related to the security aspects, namely preserving the identity of users and data security. By the very nature of cloud computing, the data belonging to the organization using a cloud service will be held in a shared environment, which is implicitly less secure than a non-shared one. In a cloud, the resources are shared between multiple tenants and the location of the resources is potentially unknown. Sensitive data or protected applications are critical for outsourcing issues. Whilst the data should be protected in a form that addresses legislative issues with respect to data location, it should at the same still be manageable by the system. Data encryption can decrease this risk besides helping to address a number of other threats that are present in a cloud computing environment.

The number and variety of applications of cloud systems with different cloud types imply the need for different security models and requirements. As such, classical authentication models may be insufficient; in fact, multi-level authentication models have to be applied. In particular, in cases of aggregation and resale of cloud systems, the mix of security mechanisms may not only lead to problems of compatibility, but may also lead to the user distrusting the model due to lack of insight. However, there are certain domains of current state of the art in cloud computing that are directly related to interoperability problem (Mezgar and Rauschecker, 2014). We highlight these domains in the remainder of this section.

Interoperability: In the world of cloud computing, interoperability means the ability to write code that works with more than one cloud provider simultaneously, regardless of the differences between the providers. Service interoperability is the ability of cloud consumers to use their data and services across multiple cloud providers with a unified management interface. Interoperability can be divided into two main groups in cloud-based systems/applications: interoperability of the cloud systems itself (cloud layer); and interoperability of the applications (Application layer - industry specific, HW/SW specific). According to IEEE experts, interoperability is even a bigger problem than security: "The greatest challenge facing longer-term adoption of cloud computing services is not security, but rather cloud interoperability and data portability...The lack of integration between these networks makes it difficult for organizations to consolidate their IT systems in the cloud and realize productivity gains and cost savings" (Weissberger, 2011).

Portability: Portability is the ability to run components or systems written for one environment in another environment. In cloud computing, this includes software and hardware environments (both physical and virtual). In this case, customers are interested to know whether they can move their data or applications across multiple cloud environments at low cost and minimal disruption. System portability allows the migration of a fully-stopped virtual machine instance or a machine image from one provider to another provider, or migrate applications and services and their contents from one service provider to another which reduces vendor lock-in problem. It is important to mention that various cloud service models (SaaS, PaaS, IaaS) may have different requirements in relation to portability and interoperability.

Data Migration: Data Migration means the periodic transfer of data from one hardware or software configuration to another or from one generation of computer technology to a subsequent generation. Migration is a necessary action for retaining the integrity of the data and for allowing users to search, retrieve, and make use of data in the face of constantly changing technology. Data migration has increasing importance as growing number of organizations store greater quantities of data in the cloud.

Interoperability and Standardization: Standardization will increase and accelerate the adoption of cloud computing as users will have a wider range of choices in cloud without vendor lock-in, with improved portability and ability to use the cloud services provided by multiple vendors. This will also include the ability to use an organization's own existing data centre resources seamlessly. There are 
two main aspects that have to be taken into account when considering standards for cloud computing. First, there are many standards from the fields of e.g. networking, shared IT systems that can be also applied in cloud computing. Second, all users tend to use the data information stored in their legacy systems. The different standards address the different interoperability issues: across cloud service types, within cloud service types and between the cloud and the enterprise. First, as cloud applications can use different types of cloud services (SaaS, PaaS, IaaS) standards are needed to define how these different services can work together. Second, within each type of cloud service (IaaS, PaaS or SaaS), open standards make it possible to avoid vendor lock-in. Third, the standards that can define how an enterprise application can communicate with cloud resources (e.g. a database) would enable that application to use cloud services directly, without modifications. Many existing IT standards contribute to the interoperability between cloud consumer applications and cloud service, and between cloud services themselves. Also, there are standardization efforts that are specifically initiated to address the interoperability issues in the cloud. Here, we refer to Open Cloud Computing Interface (OCCI), Open Grid Forum, Cloud Data Management Interface (CDMI), Storage Networking Industry Association (SNIA), IEEE P2301 - Guide for Cloud Portability and Interoperability Profiles (CPIP) (IEEE, 2015a), IEEE P2302 - Standard for Inter-cloud Interoperability and Federation (SIIF) (IEEE, 2015b). When portability is discussed, only few cloud-specific standards are referred to, related to data (Cloud Data Management Interface - CDMI) and system portability (Open Virtualization Format - OVF, IEEE P2301 Guide for Cloud Portability and Interoperability Profiles - CPIP). A more detailed list of standards can be read in (CSCC, 2014).

\subsubsection{Specific Challenges of Cloud Computing}

When taking into consideration the great number of the existing standards that could be applied in clouds and the strong and diverse requirements and demands of the different system/firms, it is obvious that the members of the IT community has to join with each other to form groups and task forces dealing with the different standardization tasks in this area (FInES, 2012). The working methodology has to consider first the existing standards and de-facto interfaces as specifications. Based on the collected materials and documents, the Reference Architectures need to be developed, to help to define and resolve the gaps in the cloud standardization space.

Cloud Computing Use Case Discussion Group (CCUCDG), Distributed Management Task Force (DMTF) and the National Institute of Standards and Technology (NIST) have developed Reference Architectures in cooperation focusing other field of cloud standardization (NIST, 2011). In the frame of most reference architectures, taxonomies for cloud components have been developed to provide a better overview on complex system structures and their operation.

Based on the collected existing standards and the demands of the customers the gap analysis has been already done (NIST, 2011). Some of the relevant standardization priorities are proposed: Standards supporting migration in all levels, Interoperability between existing in-house IT systems and cloud based systems. The detailed overview of the identified gaps is presented in (NIST, 2011).

Many of the specific challenges, related to interoperability of cloud-based systems arise from the new exciting applications in the different domains. For example, as Computer Aided Design/Manufacturing (CAD/CAM) moves into the cloud and 3D printing becomes available on-demand; soon it will become possible to simultaneously model and design products even from the convenience of a smart-phone, yet by using the computational power of the cloud. Furthermore, the integrated application of data-intensive cloud platforms and big data analytics frameworks will pose the need to collect, aggregate and process distributed real-time streaming data collected from a large number of disparate sources.

BYOD (Bring Your Own Device) solutions also can be connected to big data, social networks and cloud technology having a continuously growing importance. BYOD is a main tool for connectivity and collaboration for external and internal communication of enterprises, employees/agents who can now reach enhanced flexibility to collaborate across all platforms (interoperability requirements), through their smart devices, in real-time, 365/24/7.

As the number of smart-phone applications that will facilitate omnipresence of NG EIS is growing extensively, the long term challenge of $5 \mathrm{G}$ mobile networks will certainly need to be considered in the context of the devices interoperability. A new generation of $5 \mathrm{G}$ standards may be introduced approximately in the early 2020 (Cryderman, 2013). Since 5G aims at supporting multiple devices simultaneously, interoperability becomes its inherent challenge. 


\subsection{Interoperability Assessment in NG EIS}

Many methods are proposed in the literature to deal with interoperability assessment. Most of these methods are based on the maturity model, a framework that describes, for a specific area of interest, a number of levels of sophistication at which activities can be carried out (Alonso et al., 2010). It describes stages through which enterprises evolve in terms of interoperability and proposes plans of action in order to reach higher levels. Some comparison study has been reported in (Panetto, 2007) and (Guedria, 2008).

Generally speaking, enterprise interoperability maturity can be measured in two ways: a priori, where the measure relates to the potential of a system to be interoperable with a possible future partner whose identity is not known at the moment of evaluation, and a posteriori, where the measure relates to the compatibility measure between two (or more) known systems willing to interoperate or to the measurement of the performance of an existing interoperability relationship between two systems.

Current research on interoperability measurement mainly focuses on maturity measures based on a variety of models. There are works based on what is generally called a Maturity Model (CMMI 2013) (Guedria et al., 2015). Authors can quote LISI (Levels of Information System Interoperability) (C4ISR 1989), OIM (Organisational Interoperability Model) (Clark and Jones 1999), LCIM (Levels of Conceptual Interoperability Model) (Tolk 2003) and the new standard ISO 11354-1:2010 (ISO 2010) and CEN 11354-2:2013 (CEN 2013). Those approaches rely on qualitative but subjective assessments based on general evaluation criteria as quoted in (Panetto, 2007) and (Guedria, 2008).

\subsubsection{Specific Challenges of Interoperability Assessment}

These approaches lack formal definition of their criteria and need a high level of expertise to be applied in an industrial context (Ford et al., 2007). Some studies have tried to explore quantitative measures (Ford et al., 2007) (Daclin et al., 2008) for describing interoperability relationships, but considering the participating systems as black boxes without any concerns about the details and the semantics of the exchanged information. The approach proposed by (Castano et De Antonellis, 1998) and extended by (Bianchini et al., 2006) provides a framework for expressing and evaluating quantitatively the semantic relationships between multiple information systems. Those information systems are seen as a collection of processes that produce services to accomplish a well-defined objective. Thus, evaluating the interoperability of theses information systems is based on the evaluation of the degree of coupling between processes. This evaluation is allowed through evaluating the semantic relationships and identifying the activity replication and overlapping, as well as the aspects related to interaction/cooperation (Yahia et al., 2012).

Some significant studies have shown the usefulness of the measures precision and recall that originate from Information Retrieval (IR) (Clerverdon 1993) and have been adapted for Ontology Matching Quality (OMQ) in (Do et al., 2003) and (Ehrig and Euzenat 2005). Those measures are mainly based on the comparison of an expected result and the effective result of the evaluated system, for instance, the set of semantic relationships to be retrieved by ontology matching tools. We could not apply those measures to evaluate interoperability between information systems as they are only useful to evaluate the ability of the ontology matching tools for retrieving the right semantic relationships between information systems. Thus, precision and recall do not measure the ability of two information systems to interoperate.

\section{Towards Future Internet-based Enterprises}

According to telecom giant Cisco (2013), the Internet of Everything (IoE) is "bringing together people, process, data, and things to make networked connections more relevant and valuable than ever before, turning information into actions that create new capabilities, richer experiences, and unprecedented socio-economic and environmental opportunities for businesses and individuals". In this context, the Future Internet-based Enterprise can be defined as "an enterprise-centric network integrating and interoperating with proprietary and non-proprietary advanced technologies, computers, communication systems, control strategies as well as cognitive agents (both humans and/or advanced intelligent systems) able not only to manage processes and products, but also to generate new behaviours for adapting themselves to a dynamic market" (Davis et al., 2007). Consequently, the Future Internet-based Enterprise aims to manage and leverage on all possible networked connections among people, process, data, things and services to achieve its strategic goals. This is not possible without the system with associated capabilities, as presented in this paper. 
The networked connections have the potential to create new collaborative forms and business models between the Future Internet-based Enterprise and the IoE. In this IoE, NG EISs create, maintain and operate the digital identities of Internet-based Enterprise; each of these identities can sense and perceive the people, process, data, things and services, namely, they are aware of its environment; based on those perceptions, the identities of NG EISs can dynamically configure and re-configure to identify and use the business opportunity in a most effective and efficient way. Now, the business opportunities are drawn from the Internet of People (IoP), Internet of Processes (IoPs), Internet of Data (IoD), Internet of Things (IoT) and Internet of Services (IoS).

From a people perspective, the Internet of People (IoP), a world of smart phones and other Internetenabled personal electronics (e.g. tablets and laptops) is maturing and adding already wearable personal devices and many new human interfaces giving birth to emerging business models for industries such as health, fitness and sports for leisure and/or work. For example within the Factories of the Future (FoF), handheld computers and wearable devices will and are already starting to make possible to improve productivity and flexibility of workers and also increasing occupational safety and health at work. On the consumer goods market wearable devices and personal sensors are enabling a range of services based on measurement of physiological indicators to monitor and track the health and wellbeing of their users.

From a process perspective, the Internet of Processes (IoPs) refers to processes that go beyond typical business rules engines, these "smart" processes incorporate intelligent agent technology to build on current business process management frameworks new reasoning and adaptive capabilities that will permit business rules to learn, adapt, and act accordingly to the complexity of tasks inherent in a multiple business processes environment. For example, any task or activity within a business process can be enriched with embedded awareness data and task-appropriate analytics relevant to the business activity being executed in order to improve its overall performance. As a result, agent technology holds a great potential to provide business processes with the efficiency, flexibility, and customization that customers want from organizations today (Sinur et al., 2013), for example, from a travel agency company or an event planning service.

From a data perspective, the Internet of Data (IoD) aims to organize the data to be interconnected as a network and collect useful information for data identification, data tracing, data visualization and further data analysis, according to Wei Fan et al. (2012). The IoD concept comes from the notion that the data is meaningful only as data interoperates with other data and context can be provided to it to transform the data into information. In a world of exponential data growth, IoD explores the information about a specific data entity (metadata) using data hiding technology (Moulin and Sullivan, 2003) in order to improve the way to interconnect all the data and extract useful information from it (Wei Fan et al., 2012). For example, IoD offers a great potential to "big data" applications by proposing a new data management strategy capable of facilitating data aggregation, datasets creation, and patterns discovery to gain new or even breakthrough insights from open and/or own data. Financial services, insurance industry, marketing companies and other data-driven organizations will be able to boost their business models with big data analytic tools.

From the devices' perspective, the Internet of Things (IoT) includes tags, sensors, meters, actuators, and other types of devices that can be attached to any physical object and build a connection between the physical and digital worlds by connecting to a wired and/or wireless network to share information. The IoT is creating endless opportunities to capture in real-time basis and lower cost untapped data about products and processes to help businesses to monitor and optimize their live performance, driving as a result their organizations into full efficiency and enhanced operational performance. IoT solutions will provide as such in the coming years new organisational capabilities to achieve greater productivity, reliability, and quality of service, while reducing costs by means of real-time and even proactive improvement thanks to a continuous "Sense-Interpret-Decide-Act-Monitor" (SIDAM) operational mode that will make possible a rapid deployment model to achieve continuous and sustainable improvement. Some examples of the IoT can be already seen in manufacturing (smart factories), and distribution and logistics (smart transportation systems) processes, making their operations more intelligent and efficient, and also at products in service-enhanced products or productservice systems that rely on available information on the product to provide remote services like diagnostics and condition monitoring, support and troubleshooting, and software updates (Monostori, et al., 2015).

A fifth dimension of the IoE can be defined, named the Internet of Services (IoS), coming from a vision of "resources virtualization" and their delivery as a service. The IoS includes nowadays service models like Infrastructure-as-a-Service (IaaS), Platform-as-a-Service (PaaS), Software-as-a-Service (SaaS), and Unified Communications-as-a-Service (UCaaS). The IoS offers to their users the opportunity to build on others available resources and focus on a new strategic logic where resources 
are mobilized, managed and deployed rather than owned. As many resources become able to be virtualized, the Internet becomes a new and attractive delivery channel for such digital services.

Furthermore, the IoE is more than different Internets; it is a network of networks that aims to bring all together people, process, data, things (e.g. machines), and even services (e.g. virtual resources) in several possible combinations such as: person-to-person $(\mathrm{P} 2 \mathrm{P})$ in social networks; person-to-process (P2P) with processes visualization in models, simulators, dashboards, widgets and/or cockpits; personto-data (P2D) with semantic search accuracy; person-to-machine (P2M) with intelligent humanmachine interfaces; person-to-service (P2S) with accessibility to digital services; process-to-process (P2P) with processes mash-ups; process-to-data (P2D) with enriched datasets for data analytics; process-to-machine (P2M) with improved automation and control of machines; process-to-service (P2S) with better virtual resources availability and utilization; data-to-data (D2D) for value-added metadata; data-to-machine (D2M) with higher performance of machines due to real-time and precise data streaming; data-to-service (D2S) for better (mass-)customization of digital services; machine-tomachine (M2M) aiming a totally integrated automation and control of equipment; machine-to-service (M2S) with complementary virtual resources available on the cloud on-demand; and service-to-service (S2S) with scalable resources also available in the cloud through their virtual resources integration.

Such Internet Ecosystem described depicts the emergence of new collaborative forms between people, process, data, things, and services and calls for new "systems of systems interoperability" (SOSI) solutions within the Future Internet-based Enterprise and with the IoE, including open standards, new business models and open architectures and frameworks to support the Future Internet. These solutions constitute native architectures of NG EISs. In this context the importance of interoperability for the Future Internet Ecosystem members' from people to services is undoubted, and still from a technical and business contexts decentralized and distributed approaches for peer-to-peer networks and heterogeneous entities interoperability are still missing.

From a dual business and technical contexts, the Enterprise Interoperability Research Roadmap of the European Commission (2006) identified six dimensions which span the problem space, namely managing the rapid pace of change and innovation, adapting to globalization, change to an open innovation model, large integration and interoperability costs, difficulties in decision making when to interoperate with other entities, and lack of business case for interoperability. The latter three appear to be those directly affecting the take up of any enterprise interoperability solution, but currently based on to the emerging IoS, some of the large integration and interoperability costs have started to be reduced thanks to the appearance of "interoperability services" over the Internet. Furthermore, the decision on when to interoperate with other entities somehow has become no longer a matter of decision making but a de facto need in order to thrive, or even survive in the Future "ubiquitous" Internet and "pervasive" world driven by the IoE and all its shareholders (people, businesses, governments, etc.). Finally, more and more business cases for interoperability are emerging in the global market and society, to mention more mature global e-commerce and e-business economy that requires secure digital transactions and novel plug \& play interfaces to interoperate in an agile and flexible way within the different IoE dynamic ecosystems; new open markets of unlimited possibilities for innovative value propositions based on product-service systems and digital services enabled by the IoT and IoS, and of course the young big data analytics market enabled by the IoT and IoD.

From a pure business context, there is very limited literature available on business models for interoperability services within the scientific and practitioners' communities. Some research works have been presented by Legner \& Wende (2006), Bucherer \& Hoyer (2008), and Castro \& Alves (2011), and some practitioners' investigations by SAP (Stuhec, 2007) and Gartner (Natis, 2011). In both communities, research on business models for interoperable services is still on its infancy. An outstanding and bring-forward scientific work in this almost virgin field can be found at Otto et al. (2013) research that aims at responding to the question of how to design a business model reference for decentralized interoperability services that could enable the required SOSI development with a business sense to exploit the full business potential of the IoE. Otto et al. (2013) have presented a successful business model reference and a clear guide for reference modelling that can be used by interoperable services providers and consumers to develop and assess the value on interoperable solutions investments.

From a purely technical perspective, even that plenty of research exists on systems interoperability; the IoE challenges remain, related to highly intelligent and ubiquitous interoperability among people, process, data, things, and services. Some research works that can be highlighted in this direction are Morris et al. (2004) and DiMario (2010), both addressing the future implications for SOSI engineering. Furthermore, future SOSI solutions will be based on a combination of tight and loose coupling approaches between various systems of systems components, especially loose coupling approaches due to the ubiquitous and pervasive nature of collaboration opportunities in the IoE for interoperation 
between different entities. In this sense, Morris et al. (2004) pointed out some technology advances and common agreements that must be achieved between technology providers in order to achieve a network-centric wealth-fare for the IoE: (a) a basic research on network behaviour (emergent properties of networks), (b) network fundamentals (e.g. routing, forwarding) - adaptive dynamic networking, (c) modelling and simulation of component interactions, (d) effect of component architectures on quality attributes (e.g. security, reliability, survivability and re-configurability) of systems of systems, (e) service-oriented architectures defining basic and higher level capabilities for system composition, (f) capability to specify semantic assumptions and expectations about shared data, and (g) approaches for legacy system integration and migration.

But coming back to business context even with the necessary technology advances, success will not be achieved without corresponding changes in policy and appropriate business models and business sense incentives. DiMario (2010) introduces a holistic "social" function for systems of systems to cope with the emergence of interoperability opportunities. Such function is the global utility function whereby the system of systems, as a system, is capable of maximizing its expected utility by means of different interoperability possibilities.

The future of the Future Internet-based Enterprise is still open for many technical research and business opportunities towards a ubiquitous SOSI environment for people, process, data, things, and services to meet the full potential of the IoE.

\section{Conclusions}

This paper presents a vision statement of the IFAC Technical Committee 5.3 related to the development of NG EIS. It identifies their key properties and elaborates on some of the most important challenges for realizing these properties.

Enterprises should be addressed as complex adaptive systems, stimulated by extensive and resilient sensorial capabilities that are able to detect physical and virtual stimulus, recognizing the context of specific situations and responding and/or reacting accordingly. To achieve such capabilities, a novel framework is required with normalized reference models, formal methods, standardized architecture and open platform and tools, model and process adaptation and morphisms for data and model transformation able to realize the universal sensing capabilities of the enterprise. Actually, it is the context-aware NG EIS that will enable the enterprises with the capability for global context awareness of the business systems and with the faculty to perceive their holistic actual status in a real-time and anticipate future decisions by using multi-dimensional and multi-modal information.

The universal awareness and self-awareness of one NG EIS and its corresponding capacity to perceive the stimulus of an unknown background, character, source, underlying motivation, etc., will draw directly from a semantic interoperability as a property of this NG EIS. The Next Generation internet will be a key technological enabler of complex, loosely connected or disconnected functional networks of devices. Each of these networks will store and maintain knowledge that can be of benefit for a sensing enterprise. When this knowledge becomes available externally, the NG EIS will become capable to form on-demand virtual ecosystems of data, models, services and computational resources. Capabilities to sense these resources, perceive their meaning by combining different perceptual theories even computational methods and act upon these perceptions are crucial components of the semantic interoperability property of NG EIS.

It is foreseen that the deployment aspects of NG EIS will be mostly affected by the current research and practice in the fields of Cyber-Physical Systems and Cloud architectures.

Cyber-Physical Systems and Next Generation Internet are considered as primary deployment infrastructures for NG EIS. The further development of these paradigms will open the tremendous opportunities for realizing the presented concepts of NG EIS, but also pose the significant challenges. Some of the highlighted challenges are seamless information exchange between the cyber tools and resources across systems and platforms; and modelling issues related to concurrency, sensitivity and heterogeneity.

Cloud architectures are able to handle the great amount of data generated by sensors, smart devices and distributed, anthropocentric systems that are basic components of NG EIS. The complex cloud based systems - including e.g. BYOD, Big Data, 5G - can fulfil the demands for high-level collaboration, flexibility and dynamic behaviour set by the NG EIS. After having solved the challenges for CBSs (interoperability and lack of special standards besides security and privacy), they will become one of the basic components of the NG EIS. 
It is a fact that enterprises must collaborate, in the new environment where IT is predominant. However, enterprises, such as humans, have their own identity and their own objectives. As envisioned in this paper, NG EISs need, for seamlessly collaborating, to be dynamically adapted to the evolving environment. Thus, assessing the risk of non-functioning and their conceptual barriers, for allowing smooth interoperation between these context-aware systems is a challenging priority. Assessing, a priori, the interoperability property of these systems will help in not only focusing the development efforts to the most strategic parts of these systems, but also to develop cost-effective interfaces which are, in fact, the major driver for the Future Internet-based Enterprises in a global market.

These enterprises will be regulated open, but controlled-border cyber-physical systems of intra- and inter- systems/networks of people, process, data, things, and services cooperating towards common or compatible goals as well as competing within a Global Networked Economy. Enabling interoperability as a property of any entity in the IoE will become a technical reality in the near future, handling the dynamic categorization, membership, integration, accreditation, rewarding and disintegration of different entities within multiple networks. Nevertheless from a business perspective (economical and behavioural), interoperability research is behind in fully understanding the new "Game Theory" for business interoperability strategies and services between the multiple participating entities of the IoE. This last statement is one of the biggest challenges for the interoperability research area in the coming years.

\section{References}

1. Agievich, V., Gimranov, R., Taratoukhine, V., Becker, J. (2013). "Towards Enterprise Architecture Using Solution Architecture Models”, Enterprise Interoperability, pp. 89-94.

2. Agostinho, C., Sarraipa, J., Goncalves, D., Jardim-Goncalves, R. (2011). "Tuple-Based Semantic and Structural Mapping for a Sustainable Interoperability", Technological Innovation for Sustainability, IFIP, Advances in Information and Communication Technology, Vol. 349, pp. 4556.

3. Agostinho, C., Černý, J., Jardim-goncalves, R. (2012). "MDA-Based Interoperability Establishment Using Language Independent Information Models”, In: M. van Sinderen et al., Eds. 4th International IFIP Working Conference on Enterprise Interoperability (IWEI 2012). Harbin, China: Springer, pp. 146-160.

4. Agostinho, C., Jardim-Goncalves, R. (2015). "Sustaining Interoperability of Networked LiquidSensing Enterprises: A Complex Systems Perspective”, Annual Reviews in Control, 39(1), 128143.

5. Alonso, J., De Soria, I.M., Orue-Echevarria, L., Vergara, M. (2010). "Enterprise Collaboration Maturity Model (ECMM): Preliminary Definition and Future Challenges", Enterprise Interoperability IV, Springer Part VII, pp. 429-438.

6. Arch-int, N., Arch-int, S. (2013). "Semantic Ontology Mapping for Interoperability of Learning Resource Systems using a Rule-based Reasoning Approach”, Expert Systems with Applications, 40(18), pp. 7428-7443.

7. Bakillah, M., Liang, S. H., Zipf, A., Mostafavi, M. A. (2015). “A Dynamic and Context-Aware Semantic Mediation Service for Discovering and Fusion of Heterogeneous Sensor Data”, Journal of Spatial Information Science, (6), pp. 155-185.

8. Banerjee, A., Venkatasubramanian, K.K., Mukherjee, T., Gupta, S.K.S. (2012). "Ensuring Safety, Security, and Sustainability of Mission-Critical Cyber-Physical Systems", In Proceedings of the IEEE, Volume 100, pp. 283-299.

9. Barnaghi, P., Ganz, F., Henson, C., Sheth, A. (2012). "Computing Perception from Sensor Data", In proceedings of the 2012 IEEE Sensors Conference, Taipei, Taiwan, October 28-31.

10. Bellodi, E., Lamma, E., Riguzzi, F., Albani, S. (2011). “A Distribution Semantics for Probabilistic Ontologies", In Proceedings of URSW2011, pp. 75-86.

11. Bianchini, D., De Antonellis, V., Melchiori, M., Salvi, D. (2006). "Semantic-enriched Service Discovery", In Proceedings of the 22nd International Conference on Data Engineering Workshops ICDEW'06. IEEE Computer Society, Atlanta, GA, USA, April 2006, pp.38-48.

12. Blair, G., Grace, P. (2012). "Emergent middleware: Tackling the Interoperability Problem", IEEE Internet Computing, (1), pp. 78-82.

13. Brambilla, M., Cabot, J., Wimmer, M. (2012). "Model-driven Software Engineering in Practice", Synthesis Lectures on Software Engineering, 1(1), pp. 1-182.

14. Bucherer, E., Hoyer, V. (2008). "Business Models for Enterprise Interoperability Platforms", In Proceedings of the 14th International Conference on Concurrent Enterprising (ICE2008), Lisbon, Portugal, June 23, 2008, Centre for Concurrent Enterprises, pp. 859-866. 
15. Buyya, R. C.S., S. Yeo, J. Venugopal, Broberg, I. Brandic, (2009). "Cloud Computing and Emerging IT Platforms: Vision, Hype, and Reality for Delivering IT Services as the 5th Utility", Future Generation of Computer Systems, 25, pp. 599-616. CarTel project, http://cartel.csail.mit.edu/doku.php

16. CarTel 2015, project, http://cartel.csail.mit.edu/doku.php

17. Castano S., De Antonellis, V. (1998). "A Framework for Expressing Semantic Relationships between Multiple Information Systems for Cooperation”, Information Systems, 23(3-4), pp. 253277.

18. Castro, H. Alves, A.P. (2011). “A P2P Content Delivery System for Alternative Business Models Harnessing Internet's Full Potential”, In Proceedings of the 7th International Conference on Next Generation Web Services Practices (NWeSP 2011), Salamanca, Spain, 2011, pp. 86-92.

19. Cecil, J., Ramanathan, P., Rahneshin, V., Prakash, A., Pirela-Cruz, M. (2013). "Collaborative Virtual Environments for Orthopedic Surgery", In Proceedings of the 9th annual IEEE International Conference on Automation Science and Engineering (IEEE CASE 2013), August 17 to 21, Madison, WI.

20. Cecil, J, Jones, J. (2014). “An Advanced Virtual Environment for Micro Assembly, International Journal of Advanced Manufacturing Technology", 72(1) pp. 47-56

21. Cecil, J. (2015) US IGNITE: A Gigabit Network and Cyber-Physical Framework for Advanced Manufacturing", NSF Computer and Networking Systems, https://vrice.okstate.edu/content/gigabit-network-and-cyber-physical-framework

22. CEN TS 11354-2. "Maturity Model for Assessing Enterprise Interoperability", Geneva, 2013

23. CISCO (2013). "Internet of Everything At-A-Glance", http://www.cisco.com/web/about/ac79/docs/IoE/IoE-AAG.pdf

24. Chen, D., Daclin, N. (2007). "Barriers Driven Methodology for Enterprise Interoperability in Establishing the Foundation of Collaborative Networks", IFIP TC 5 WG 5.5, In Proceedings of 8th IFIP Working Conference on Virtual Enterprises, Volume 243, Guimaraes, Portugal, pp. 453-460.

25. Clark T., Jones, R. (1999). "Organisational Interoperability Maturity Model for C2", In Proceedings of the Command and Control Research and Technology Symposium (CCRTS), Newport, RI.

26. Cleverdon, C., (1993). "The Cranfield tests on index language devices", In MCB UP Ltd, pp.173-194.

27. CMMI 1.3 (2013). “Capability Maturity Model Integration”, SEI, Carnegie Mellon University.

28. Compton, M., Henson, C., Lefort, L., Neuhaus, H., Sheth, A. (2009). "A Survey of the Semantic Specification of Sensors", In Proceedings of 2nd International Semantic Sensor Networks Workshop.

29. Compton, M., Barnaghi, P., Bermudez, L., Garcia-Castro, R., Corcho, O., Cox, S., Graybeaf, J., Hauswirth, M., Henson, C., Herzog, A., Huang, V., Janowitz, K., Kelsey, D., Le Phuoc, D., Lefort, L., Leggieri, M., Neuhaus, H., Nikolov, A., Page, K., Passant, A., Sheth, A., Taylor, K. (2012). "The SSN Ontology of the W3C Semantic Sensor Network Incubator Group", Journal of Web Semantics: Science, Services and Agents on the World Wide Web, 17, pp. 25-32.

30. Costa, P.C.G., Laskey, K.B. (2006). "PR-OWL: A Framework for Probabilistic Ontologies", In Proceedings of the 2006 conference on Formal Ontology in Information Systems: Proceedings of the 4th International Conference (FOIS 2006), pp. 237-249. IOS Press Amsterdam.

31. Cretan, A., Coutinho, C., Bratu, B., Jardim-Goncalves, R. (2012). "NEGOSEIO: A Framework for Negotiations toward Sustainable Enterprise Interoperability”, Annual Reviews in Control, 36(2), pp. 291-299.

32. Cryderman, J. (2013). "5G and the Future of Wireless Networks", 10(5) http://www.pipelinepub.com/network_evolution/5G

33. C4ISR, Interoperability Working group C4ISR, "Levels of Information Systems Interoperability (LISI)", US Department of defense, Washington, DC, Architectures Working Group report February 1998.

34. Chungoora, N., Young, R. I., Gunendran, G., Palmer, C., Usman, Z., Anjum, N. A., ... Case, K. (2013). "A Model-Driven Ontology Approach for Manufacturing System Interoperability and Knowledge Sharing”, Computers in Industry, 64(4), pp. 392-401.

35. CSCC, Cloud Standards Customer Council (CSCC). (2014) "Interoperability and Portability for Cloud Computing: A Guide" http://www.cloud-council.org/CSCC-Cloud-Interoperability-andPortability.pdf

36. Daclin N., Chen, D., Vallespir, B (2008). "Methodology for Enterprise Interoperability", In Proceedings of the 17th World Congress: The International Federation of Automatic Control, Seoul, Korea, pp. 6-11. 
37. Danila, C., Stanescu, A., Jardim-Goncalves, R., Agostinho, C., Florea, A.M., Serbanescu, K. (2013), "Sustainable Interoperability of Sensing Enterprise", In Proceedings of the IFAC Conference on Manufacturing Modelling, Management, and Control, St. Petersburg, Russia, pp.1304-1309.

38. Davis, J. P., Eisenhardt, K., Bingham, B. C. (2007). "Complexity Theory, Market Dynamism and the Strategy of Simple Rules", In Proceedings of DRUID Summer Conference 2007 on Appropriability, Proximity Routines and Innovation, Copenhagen, CBS, Denmark, June 18-20.

39. Derler, P., Lee, E.A., Vincentelli, A.S. (2012). "Modelling Cyber-Physical Systems", In Proceedings of the IEEE, 2012, Volume 100, pp. 13-28.

40. Diallo, S. Y., Tolk, A., Graff, J., \& Barraco, A. (2011). "Using the Levels of Conceptual Interoperability Model and Model-based Data Engineering to develop a Modular Interoperability Framework", In Simulation Conference (WSC), Proceedings of the 2011 Winter, pp. 2571-2581, IEEE.

41. DiMario, M.J. (2010). "System of Systems Collaborative Formation”, World Scientific Publishing Co. Pte. Ltd., ISBN: 13 978-981-4313-88-9.

42. Ding, Z., Peng, Y. (2004). "A Probabilistic Extension to Ontology Language OWL", In Proceedings of the 37th Annual Hawaii International Conference on System Sciences, pp. 5-8.

43. Distributed Robotics Garden, http://groups.csail.mit.edu/drl/wiki/index.php?title=The_Distributed_Robotics_Garden

44. Do, H.H., Melnik, S., Rahm, E. (2003). "Comparison of Schema Matching Evaluations", WebServices, and Database Systems, Lecture Notes in Computer Science Volume 2593, pp 221-237.

45. Doan, A., Noy, N.F., Halevy, A.Y. (2004). "Introduction to the Special Issue on Semantic Integration", ACM SIGMOD Record, 33(4), pp. 11-13.

46. Ducq, Y., Chen, D., Alix, T. (2012). "Principles of Servitization and Definition of an Architecture for Model Driven Service System Engineering", In Proceedings of the 4th International IFIP Working Conference on Enterprise Interoperability (IWEI 2012). Harbin, China: Springer.

47. Ducq, Y., Chen, D., Doumeingts, G. (2012). "A Contribution of System Theory to Sustainable Enterprise Interoperability Science Base”, Computers in Industry, 63(8), pp. 844-857.

48. ECIS 15, “Openness and IT", http://www.ecis2015.eu/participation/list-tracks/23-openness-and-it

49. Ehrig M., Euzenat, J. (2005). "Relaxed Precision and Recall for Ontology Matching", In Proceedings of Integrating Ontologies Workshop, Banff, Canada.

50. Enikov, E., Lazarov, K. (2001). "Optically Transparent Gripper for Microassembly", In Proceedings of SPIE Microrobotics and Microassembly III, Volume 4568, pp. 40-49.

51. EU FIRE Initiative, http://www.ict-fire.eu/getting-started/why-future-internet-research.html

52. European Commission (2006). "Enterprise Interoperability: A Concerted Research Roadmap for Shaping Business Networking in the Knowledge-based Economy", http://cordis.europa.eu/ist/ictent-net/ei-roadmap_en.htm

53. Euzenat, J. (2001). "Towards a Principled Approach to Semantic Interoperability", In Proceedings of IJCAI 2001 workshop on ontology and information sharing, Seattle.

54. Fan, W., Chen, Z., Xiong, Z., Chen, H. (2012). "The Internet of Data: A New Idea to Extend the IOT in the Digital World", Frontiers of Computer Science, 6(6), pp. 660-667.

55. Ferreira, J., Beca, M.F., Agostinho, C., Nunez, M.J., Jardim-Goncalves, R. (2013). "Standard Blueprints for Interoperability in Factories of the Future (FoF)", In proceedings of the 7th IFAC Conference on Manufacturing Modelling, Management and Control, St. Petersburg, Russia, pp. 1322-1327.

56. FInES, a. FInES Cluster, "Position Paper on Orientations for FP8", http://cordis.europa.eu/fp7/ict/enet/documents/fines-position-paper-fp8-orientations-final.pdf

57. FInES, b. "Future Internet Enterprise Systems (FInES) Research Roadmap 2025", http://cordis.europa.eu/fp7/ict/enet/documents/fines-research-roadmap-v30_en.pdf

58. FInES (2012). "Future Internet Enterprise Systems (FInES) Standardisation Task Force Report", Working DRAFT, (Second Edition), 16 March.

59. Fisher, D.A. (2006). "An Emergent Perspective on Interoperation in Systems of Systems", Carnegie-Mellon University of Pittsburgh, Software Engineering Institute, Technical Report CMU/SEI-2006-Tr-003, ESC-Tr-2006-003, 2006.

60. Ford T., Colombi, J., Graham, S., Jacques, D. (2007). "The Interoperability Score", In Proceedings of the 5th Conference on Systems Engineering Research, Hoboken, NJ, USA, March 14-16.

61. Fortineau, V., Paviot, T., \& Lamouri, S. (2013). "Improving the Interoperability of Industrial Information Systems with Description Logic-based Models-The State of the Art", Computers in Industry, 64(4) pp. 363-375. 
62. Fox, M.S., Barbuceanu, M., Gruninger, M. (1996). “An Organization Ontology for Enterprise Modelling: Preliminary Concepts for Linking Structure and Behaviour", Computers in Industry, 29(1-2), pp. 123-134.

63. Frankston, B. (2009). "Ambient Connectivity: An Introduction", http://frankston.com/public/?n=IAC

64. GENI, Global Environments for Network Innovation (GENI), http://www.geni.net

65. Gangemi, A., Guarino, N., Masolo, C., Oltramari, A. Schneider, L. (2002). "Sweetening Ontologies with DOLCE", Knowledge Engineering and Knowledge Management: Ontologies and the Semantic Web. Lecture Notes in Computer Science Volume 2473, pp. 166-181.

66. Gangemi, A. (2005). “Ontology Design Patterns for Semantic Web Content”, Lecture Notes in Computer Science, Volume 3729, pp. 262-276.

67. Gharajedaghi, J. (2011). "Systems Thinking: Managing Chaos and Complexity: A Platform for Designing Business Architecture", Elsevier.

68. Gilad, B. (2008). "The Future of Competitive Intelligence: Contest for the Profession's Soul", Competitive Intelligence Magazine, 11(5), p. 22.

69. Gobinath, N., Cecil, J. (2005). "Development of a Virtual and Physical Work Cell to Assemble Micro-devices", International Conference on Flexible Automation and Intelligent Manufacturing, Volume 21, pp. 431-441.

70. Gruber, T.R. (1991). "The Role of Common Ontology in Achieving Sharable, Reusable Knowledge Bases", In Principles of Knowledge Representation and Reasoning: Proceedings of the Second International Conference, pp. 601-602, Morgan Kaufmann.

71. Guarino, N. (1997). "Understanding, Building and Using Ontologies", International Journal of Human-Computer Studies, 46 (2-3), pp. 293-310.

72. Guédria W., Naudet, Y., Chen, D. (2008). "Interoperability Maturity Models - Survey and Comparison", In Proceedings of $3^{\text {th }}$ International Workshops on Enterprise, Interoperability and Networking (EI2N 2008). OTM Confederated International Workshops and Posters, Monterrey, Mexico, LNCS 5333, Springer, pp. 273-282.

73. Guédria, W., Naudet, Y., Chen, D. (2015) "Maturity Model for Enterprise Interoperability", Enterprise Information Systems, 9(1), pp. 1-18.

74. Henson, C., Thirunarayan, K., Sheth, A. (2011). "An Ontological Approach to Focusing Attention and Enhancing Machine Perception on the Web", Applied Ontology Journal, 6(4) pp. 345-376.

75. Horrocks, I., Patel-Schneider, P.F., Boley, H., Tabet, S., Grosof, B., Dean, M. (2004). "SWRL: A Semantic Web Rule Language - Combining OWL and RuleML", W3C Member Submission.

76. IEEE (2015a). "IEEE P2301 - Guide for Cloud Portability and Interoperability Profiles (CPIP)", http://standards.ieee.org/develop/project/2301.html.

77. IEEE, (2015b). "IEEE P2302 - Standard for Intercloud Interoperability and Federation (SIIF)", http://standards.ieee.org/develop/project/2302.html.

78. INCOSE, T.O., 2007. "Systems Engineering Vision 2020" (INCOSE-TP-2004-004-02), http://www.incose.org/ProductsPubs/pdf/SEVision2020_20071003_v2_03.pdf

79. ISO FDIS 11354-1, “Advanced Automation Technologies and their Applications-Requirements for Establishing Manufacturing Enterprise Process Interoperability - Part 1: Framework for Enterprise Interoperability", ISO, Geneva, Switzerland, TC 184/SC 52010.

80. Jardim-Goncalves, R., Grilo, A., Agostinho, C., Lamphataki, F., Charalabidis, Y. (2013). "Systematisation of Interoperability Body of Knowledge: the foundation for Enterprise Interoperability as a Science”, Enterprise Information Systems, 7(1), pp. 7-32.

81. Jardim-Goncalves,R., Grilo, A., Popplewell, K. (2012). "Sustainable Interoperability: The Future of Internet based Industrial Enterprises", Computers in Industry, 63(8), pp. 731-738.

82. Jardim-Goncalves, R., Agostinho, C., Steiger, A. (2011). "A Reference Model for Sustainable Interoperability in Networked Enterprises: Towards the Foundation of EI Science Base", In Proceedings of IJCIM.

83. Jardim-Goncalves, R., Grilo, A. (2010). "Building Information Modelling and Interoperability", Automation in Construction.

84. JRC. "Envisioning Digital Europe 2030: Scenarios for ICT in Future Governance and Policy Modelling”, JRC European Commission, http://ftp.jrc.es/EURdoc/JRC61593.pdf

85. Juarez, J., Rodriguez-Mondejar, J. A., Garcia-Castro, R. (2014). "An Ontology-driven Communication Architecture for Spontaneous Interoperability in Home Automation Systems", In Emerging Technology and Factory Automation (ETFA), IEEE, pp. 1-4.

86. Kalfoglou, Y., Schorlemmer, M. (2004). "Formal Support for Representing and Automating Semantic Interoperability", The Semantic Web: Research and Applications. Lecture Notes in Computer Science, Volume 3053, pp. 45-60. 
87. Kanduri, A., Rahmani, A-M., Liljeberg, P., Wan, K., Man, K.L., Plosila, J. (2013). “A Multicore Approach to Model-Based Analysis and Design of Cyber-Physical Systems", In Proceedings of ISOCC 2013, http://www.bpti.lt/uploads/Publikacijos/SS5-5-0334-F.pdf

88. Kattenstroth, H., May, W., Schenk, F. (2007). "Combining OWL with F-Logic Rules and Defaults", In Proceedings of the ICLP'07 Workshop on Applications of Logic Programming to the Web, Semantic Web and Semantic Web Services, ALPSWS 2007, Porto, Portugal, September 13 th.

89. Kyoung-Dae, K., Kumar, P.R. (2012). "Cyber-Physical Systems: A Perspective at the Centennial”, In Proceedings of the IEEE, Volume 100, Issue 1, pp. 1287-1308.

90. Kwei-Jay, L., Panahi, M. (2010). "A Real-Time Service-oriented Framework to Support Sustainable Cyber-Physical Systems", In: Proceedings of 8th IEEE International Conference on Industrial Informatics (INDIN), pp. 15-21.

91. Lee, J., Madnick, S., Siegel, M. (1996). “Conceptualizing Semantic Interoperability: A Perspective from the Knowledge Level”, International Journal of Cooperative Information Systems. 5(4), pp. 367-394.

92. Legner, C., Wende, K. (2006). "Towards an Excellence Framework for Business Interoperability", In: Proceedings of the 19th Bled eConference, Bled, Slovenia.

93. Lezoche, M., E. Yahia, A. Aubry, H. Panetto, M. Zdravković (2012). "Conceptualising and Structuring Semantics in Cooperative Enterprise Information Systems Models", Computers in Industry, 63(8), pp. 775-787.

94. Liao, Y., Lezoche, M., Panetto, H., Boudjlida, N., Loures, E. R. (2015). "Semantic Annotation for Knowledge Explicitation in a Product Lifecycle Management Context: A Survey", Computers in Industry, Volume 71, Issue 1, pp. 24-34.

95. Littlejohn, S.W., Foss, K.A. (2010). "Theories of Human Communication”, Waveland Press, Inc.

96. Madni, A.M., Lin, W., Madni, C.C. (2001). "IDEONTM: An Extensible Ontology for Designing, Integrating and Managing Collaborative Distributed Enterprises", Systems Engineering, 4(1), pp. $35-48$.

97. Mayyas, M., Zhang, P., Lee, W. H., Popa, D., Chiao, J. C. (2009). “An Active Micro Joining Mechanism for 3D Assembly", Journal of Micromechanics and Microengineering, 19(3), pp. 1-12.

98. Mell P. and T. Grance, (2009), "The NIST Definition of Cloud Computing”, Version 15, 10-7-09 from http://www.nist.gov/itl/cloud/upload/cloud-def-v15.pdf

99. Memon, M., Wagner, S. R., Pedersen, C. F., Beevi, F. H. A., Hansen, F. O. (2014). “Ambient Assisted Living Healthcare Frameworks, Platforms, Standards, and Quality Attributes", Sensors, 14(3), pp. 4312-4341.

100.Mezgar, I., Rauschecker, U. (2014). "The Challenge of Networked Enterprises for Cloud Computing Interoperability", Computers in Industry, 65(4), pp. 657-674.

101. Middleton, P. (2013). "Gartner Says the Internet of Things Installed Base Will Grow to 26 Billion Units By 2020”, STAMFORD, Conn., View All Press Releases, http://www.gartner.com/newsroom/id/2636073

102.Minnick, J. (2013). "Benefits of Cloud Computing", http://www.mbtmag.com/articles/2013/05/how-manufacturers-can-benefit-cloud-computing

103.Mokhtar, S. B., Raverdy, P. G., Urbieta, A., Cardoso, R. S. (2012). "Interoperable Semantic and Syntactic Service Discovery for Ambient Computing Environments", Innovative Applications of Ambient Intelligence: Advances in Smart Systems: Advances in Smart Systems, Volume 213.

104.Monfort, V., Cherif, S. (2012). "Bridging the Gap between Technical Heterogeneity of ContextAware Platforms: Experimenting a Service Based Connectivity between Adaptable Android", WComp and OpenORB. arXiv preprint arXiv:1203.0400.

105.Monostori, L., Valckenaers, P., Dolgui, A., Panetto, H., Brdys, M., Csáji, B. C. (2015). "Cooperative Control in Production and Logistics", Annual Reviews in Control, Volume 39, Issue 1, pp. 12-29.

106.Morris, E., Levine, L, Meyers, C., Place, P, Plakosh, D. (2004). "System of Systems Interoperability (SOSI)", Technical Report CMU/SEI-2004-TR-004, ESC-TR-2004-004, Integration of Software-Intensive Systems Initiative, Carnegie Mellon Software Engineering Institute.

107.Motik, B., Horrocks, I., Rosati, R., Sattler, U. (2006). "Can OWL and Logic Programming Live together Happily Ever After?”, Lecture Notes in Computer Science, Volume 4273, pp. 501-514, In: Proceedings of 5th International Semantic Web Conference, ISWC 2006, Athens, GA, USA, November 5-9.

108. Moulin P., O’Sullivan J. A. (2003). "Information-Theoretic Analysis of Information Hiding”, IEEE Transactions on Information Theory, 49(3), pp. 563-593. 
109.Natis, Y.V. (2001). "Hype Cycle for Cloud Application Infrastructure Services (PaaS)", Gartner, Stamford, CT.

110.Naudet, Y., Latour, T., Guedria, W., Chen, D. (2010). "Towards a Systemic Formalisation of Interoperability", Computers in Industry. 61(2), pp. 176-185.

111.NIST, 2011. Special Publication 500-291, NIST Cloud Computing Standards Roadmap, July (2011).

112.Neisser, U. (1976). "Cognition and Reality", Psychology Volume 218, San Francisco: W.H. Freeman and Company.

113.Nof, S.Y., Morel, G., Monostori, L., Molina, A., Filip, F. (2006). "From Plant and Logistics Control to Multi-Enterprise Collaboration: Milestone Report of the Manufacturing \& Logistics Systems Coordinating Committee", Annual Reviews of Control, 30(1), pp. 55-68.

114.Noy, N.F. (2004). "Semantic Integration: A Survey of Ontology-based Approaches", ACM SIGMOD Record, 33(4), pp. 65-70.

115.Otto, B., Ebner, V., Baghhi, E., Bittmann, R. (2013). "Toward a Business Model Reference for Interoperability Services", Computers in Industry, 64(8) pp. 887-897.

116.Panetto, H., J. Cecil (2013). "Information Systems for Enterprise Integration, Interoperability and Networking: Theory and Applications", Enterprise Information Systems, 7(1), pp. 1-6.

117.Panetto, H., Molina, A. (2008). "Enterprise Integration and Interoperability in Manufacturing Systems: Trends and Issues", Computers in Industry, 59(7), pp. 641-646.

118.Panetto, H. (2007). "Towards a Classification Framework for Interoperability of Enterprise Applications", International Journal of Computer Integrated Manufacturing, 20(8), pp. 727-740.

119.Perera, C., Zaslavsky, A., Liu, C. H., Compton, M., Christen, P., Georgakopoulos, D. (2014). "Sensor Search Techniques for Sensing as a Service Architecture for the Internet of Things", Sensors Journal, IEEE, 14(2), pp. 406-420.

120.Rezaei, R., Chiew, T. K., Lee, S. P., Aliee, Z. S. (2014). “A Semantic Interoperability Framework for Software as a Service Systems in Cloud Computing Environments", Expert Systems with Applications, 41(13), pp. 5751-5770.

121.Reggia, J. A., Peng, Y. (1987). "Modelling Diagnostic Reasoning: A Summary of Parsimonious covering Theory", Computer Methods and Programs in Biomedicine, 25(2), pp. 125-134.

122.Santucci, G., Martinez, C., Vlad-câlcic, D. (2012). "The Sensing Enterprise”, In proceedings of FInES Workshop at FIA 2012, Aalborg, Denmark. http://www.theinternetofthings.eu/sites/default/files/\%5Buser-name\%5D/Sensing-enterprise.pdf

123.Shadbolt, N., Berners-Lee, T. (2008). "Web Science Emerges", Scientific American, 299(4), pp.76-81.

124.Sinur, J., Odell, J. J., Fingar, P. (2013). "Business Process Management: The Next Wave", Meghan-Kiffer Press.

125.Sowa, J. (2000). "Knowledge Representation: Logical, Philosophical, and Computational Foundations", CA:Brooks/Cole Publishing Co.

126.Svejvig, P. and Møller, C. (2012). "A Workshop about the Future of Enterprise Information Systems", Re-conceptualizing Enterprise Information Systems, Lecture Notes in Business Information Processing, Volume 105, pp. 45-57.

127.Stuhec, G. (2007). "Using CCTS Modeler Warp 10 to Customize Business Information Interfaces", SAP.

128.Sztipanovits, J., Koutsoukos, X., Karsai, G., Kottenstette, N., Antsaklis, P., Gupta, V., Goodwine, B., Baras, J., Shige, W. (2012). "Toward a Science of Cyber-Physical System Integration", In Proceedings of the IEEE, Volume 100, pp. 29-44.

129.Tolk, A. (2003). "Beyond Technical Interoperability - Introducing a Reference Model for measures of merit for coalition interoperability", In Proceedings of the 8th International Command and Control Research and Technology Symposium (ICCRTS), Washington, DC, June 17-19, 2003.

130.Uschold, M., King, M., Moralee, S., Zorgios, Y. (1998). “The Enterprise Ontology”, Knowledge Engineering Review, 13(1), pp. 31-89.

131.USIgnite, What is US Ignite, http://us-ignite.org/

132. Vermesan, O., Friess, P. (Eds.). (2013). "Internet of Things: Converging Technologies for Smart Environments and Integrated Ecosystems", River Publishers.

133.Vicaire, P.A., Hoque, E., Zhiheng, X., Stankovic, J.A. (2012). "Bundle: A Group-Based Programming Abstraction for Cyber-Physical Systems", IEEE Transactions on Industrial Informatics, 8(2), pp. 379-392.

134.Villegas, N. M. (2013). “Context Management and Self-Adaptivity for Situation-Aware Smart Software Systems" (Doctoral dissertation, University of Victoria). 
135.Völter, M., Stahl, T., Bettin, J., Haase, A., Helsen, S. (2013). "Model-driven Software Development: Technology, Engineering and Management”, John Wiley \& Sons.

136.W3C (2011). "Semantic Sensor Network XG Final Report", W3C Incubator Group Report 28 June 2011, http://www.w3.org/2005/Incubator/ssn/XGR-ssn-20110628/

137.Wang, X., Xu, X., Li, W., Mehnen, J. (Eds.) (2013). "ICMS: A Cloud-Based Manufacturing System", Cloud Manufacturing, Springer series in Advanced Manufacturing, pp. 1-22, Springer Verlag, London.

138.Wang, X., Xu, X. (2013) “An Interoperable Solution for Cloud Manufacturing”, Robotics and Computer-Integrated Manufacturing, 29(4), pp. 232-247.

139.Wang, X., Xu, X. (2013) "Virtual Function Block Mechanism in the Cloud Manufacturing Environment", Advanced Materials Research, Volume 2438, pp. 694-697.

140.Weissberger, A. (2011) "IEEE CIO Says Cloud Interoperability a Bigger Problem than Security!", http://community.comsoc.org/blogs/alanweissberger/ieee-cio-says-cloud-interoperability-biggerproblem-security

141.Whitman L., Santanu, D., Panetto, H. (2006). “An Enterprise Model of Interoperability”, In Proceeding of the 12th IFAC Symposium on Information Control Problems in Manufacturing, Volume 12, Saint Etienne, France, IFAC-PapersOnline.

142.Woochul, K., Kapitanova, K., Hyuk, S.S. (2012). "RDDS: A Real-Time Data Distribution Service for Cyber-Physical Systems", IEEE Transactions on Industrial Informatics, Volume 8, pp.393-405.

143.Wu, C.-H., Chuang, Z.-J., Lin, Y.-C. (2006). "Emotion Recognition from Text Using Semantic Labels and Separable Mixture Models", ACM Transactions on Asian Language Information Processing, 5(2), pp. 165-183.

144. Yahia, E., Aubry, A., Panetto, H. (2012). "Formal Measures for Semantic Interoperability Assessment in Cooperative Enterprise Information Systems", Computers in Industry, 63(5), pp. 443-457.

145.Zhang, W., Hansen, K. M., \& Bellavista, P. (2013). "A Research Roadmap for ContextAwareness-based Self-managed Systems", In Service-Oriented Computing-ICSOC 2012 Workshops, pp. 275-283, Springer Berlin Heidelberg.

146.Zdravković, M., Panetto, H., Trajanović, M., Aubry, A. (2011). “An Approach for Formalising the Supply Chain Operations”, Enterprise Information Systems, 5(4), pp. 401-421.

147.Zdravković, M., Noran, O., Panetto, H., Trajanović, M. (2015). "Enabling Interoperability as a Property of Ubiquitous Systems for Disaster Management”, Computer Science and Information Systems, In Press. DOI 10.2298/CSIS141031011Z

148.Zdravković, M., Trajanović, M., Panetto, H., Aubry, A. (2014a). "Explication and Semantic Querying of Enterprise Information Systems", Knowledge and Information Systems, 40(3), pp. 697-724.

149.Zdravković, M., Noran, O., Trajanović, M. (2014b). "Towards Sensing Information Systems”, In proceedings of the 23rd International Conference on Information Systems Development, Varaždin, Croatia, September 2-4. 
Appendix 1: Overview of Existing Body of Knowledge: Current Issues and Foreseen Solutions for the Challenges of Interoperable NG EIS

\begin{tabular}{|c|c|c|c|}
\hline & $\begin{array}{c}\text { Existing Resources } \\
\text { (Theories, Models, Languages, Tools, ...) }\end{array}$ & $\begin{array}{l}\text { Key Current Problems } \\
\text { (Research Gaps) }\end{array}$ & $\begin{array}{c}\text { Envisaged Solutions with a Timeframe } \\
\text { (Short, Medium, Long-term) }\end{array}$ \\
\hline Context-Aware Systems & $\begin{array}{l}\text { - Cyber-Physical Systems (CPSs) } \\
\text { - Systems complexity } \\
\text { - Model-based Systems } \\
\text { - Service engineering } \\
\text { - Conformance testing } \\
\text { - Interoperability checking }\end{array}$ & $\begin{array}{l}\text { - Dynamic configurability } \\
\text { - Competitive and Customer Intelligence } \\
\text { - Intelligent reconfiguration } \\
\text { - Automatized categorization of ontological structures } \\
\text { - Transient analysis } \\
\text { - Adaptive Services }\end{array}$ & $\begin{array}{l}\text { - IoT-facilitated intelligent response of the enterprises } \\
\text { to their environment (short). } \\
\text { - Implementing virtual and physical sensing } \\
\text { capabilities and connecting them to the digital } \\
\text { capabilities of the Future Internet (medium). } \\
\text { - Inclusive (multiple dimensions and stakeholders) } \\
\text { sensing (long). } \\
\text { - Consolidation of efforts to create large possibilities } \\
\text { of innovative business. (long). }\end{array}$ \\
\hline Cloud-based systems & $\begin{array}{l}\text { - Deployment models for the different } \\
\text { business cases, } \\
\text { - Service models for the different user } \\
\text { demands, } \\
\text { - Virtual machines for data handling. }\end{array}$ & $\begin{array}{l}\text { - Different, complex types of interoperability } \\
\text { between/inside clouds and conventional systems. } \\
\text { - Lack of standards for CC special interoperability } \\
\text { problems. }\end{array}$ & $\begin{array}{l}\text { - International, structured efforts to classify existing } \\
\text { usable interoperability standards for cloud (short). } \\
\text { - Define needs and specifications of new cloud } \\
\text { standards (medium). } \\
\text { - Continuous development of standards (long). }\end{array}$ \\
\hline $\begin{array}{l}\text { Enterprises as } \\
\text { Cyber-Physical systems }\end{array}$ & $\begin{array}{l}\text { - Information Centric models for } \\
\text { collaboration. } \\
\text { - Next Generation Internet Test Beds. } \\
\text { - Cloud based technologies serve as } \\
\text { enables of CPS. }\end{array}$ & $\begin{array}{l}\text { - Seamless data/information exchange techniques } \\
\text { integrating use of sensory and other data with cyber } \\
\text { tools across systems and platforms. } \\
\text { - Ultra-fast network with high gigabit capability. } \\
\text { - Modelling cyber-physical systems is emphasized } \\
\text { including concurrency, sensitivity and heterogeneity. }\end{array}$ & $\begin{array}{l}\text { - Global consortium with focused industry efforts (in } \\
\text { medicine/healthcare, manufacturing, transportation, } \\
\text { education, energy systems, among others). } \\
\text { - Innovative Education and Curriculum development } \\
\text { addressing industry needs. }\end{array}$ \\
\hline $\begin{array}{l}\text { Interoperability } \\
\text { Assessment }\end{array}$ & $\begin{array}{l}\text { - Capability Maturity models } \\
\text { - Semantics formalisation } \\
\text { - Ontology engineering and reuse } \\
\text { - Formal measures } \\
\text { - Concepts mapping and matching } \\
\text { - Formal Concepts Analysis (FCA) }\end{array}$ & $\begin{array}{l}\text { - Lack of formal maturity models. } \\
\text { - Lack of practical/automatized concepts mapping tools. } \\
\text { - Size/scale of existing models and ontology. }\end{array}$ & $\begin{array}{l}\text { - Definition of a maturity model based on a priori and } \\
\text { a posteriori semantics measures (short). } \\
\text { - Generic automatic semantic matching tool (long). } \\
\text { - Natural Language Processing tools (long). } \\
\text { - Data mining tools (medium). } \\
\text { - Formal interoperability theory (medium). }\end{array}$ \\
\hline Semantic Interoperability & $\begin{array}{l}\text { - Ontology engineering methodology } \\
\text { - Ontology engineering tools (e.g. Protégé) } \\
\text { - Semantic annotation } \\
\text { - Manual semantic integration } \\
\text { - Sensor ontologies } \\
\text { - Dolce Upper ontology } \\
\text { - Relatively high number of different } \\
\text { domain ontologies. } \\
\text { - Transformation tools (e.g. database-to- } \\
\text { ontology, UML-to-ontology). }\end{array}$ & $\begin{array}{l}\text { - Lack of unified interoperability theory. } \\
\text { - Fragmented and uncorrelated ontologies. }\end{array}$ & $\begin{array}{l}\text { - Development and validation of the Formal } \\
\text { interoperability theory (short). } \\
\text { - Establishing commitment to "Human" ontology } \\
\text { continuum methodology (short). } \\
\text { - Development of Ontology engineering frameworks } \\
\text { (based on the formal interoperability theory and } \\
\text { methodology) (medium). } \\
\text { - Further integration and consolidation of work on } \\
\text { combining deterministic and non-deterministic } \\
\text { logics (long). }\end{array}$ \\
\hline
\end{tabular}

